\title{
Localized Propagating Tachyons in Extended Relativity Theories
}

\author{
Matej Pavšič \\ Jožef Stefan Institute, Jamova 39, 1000 Ljubljana, Slovenia \\ e-mail: matej.pavsic@ijs.si
}

\begin{abstract}
We examine the possibility of localized propagating tachyonic fields within a properly extended relativity. A possible extension is to include superluminal transformations and reference frames. This leads to complex $4 D$ spacetime, or real $8 D$ spacetime $M_{4,4}$. The mass shell constraint in $M_{4,4}$ becomes, after first quantization, the ultrahyperbolic KleinGordon equation. The Cauchy problem for such equation is not well posed, because it is not possible to freely specify initial data on a $7 \mathrm{D}$ hypersurface of $M_{4,4}$. We explicitly demonstrate that it is possible to do it on a space-like 4-surface for bradyons, and on a time-like 4-surface for tachyons. But then the evolution of a bradyonic field into the four time-like directions, or the "evolution" of a tachyonic field into the four space-like directions, is not uniquely determined. We argue that this is perhaps no so bad, because in quantum field theory (after second quantization) the classical trajectories of fields are not determined anyway, and so it does not matter, if they are not completely determined already in the first quantized theory. A next possible extension of relativity is to consider $16 D$ Clifford space, $C$, a space whose elements are oriented $r$-volumes, $r=0,1,2,3,4$ of real $4 D$ spacetime. Then the evolution parameter can be associated with an extra light-cone coordinate, e.g., with the sum of the scalar and the pseudoscalar coordinate, and initial data can be given on a light-like hypersurface, in which case the Cauchy problem is well posed. This procedure brings us to the Stueckelberg theory which contains localized propagating tachyons in $4 D$ spacetime.
\end{abstract}

Keywords: Tachyons, extra dimensions, Clifford space, Clifford algebras, Stueckelberg theory, tachyonic Dirac equation, ultrahyperbolic field equations, localizability, Cauchy problem, Causality, Everett interpretation

\section{Introduction}

Recent experimental results [1] have revived the interest in superluminal particles, the so called tachyons. Later, an error was found in that experiment, but this fact itself does not exclude the possibility of the existence of tachyons (to be eventually detected in some other experiment), because the theory of relativity can be suitably adapted or extended so to admit such particles [2]-9]. The possibility of superluminal electromagnetic waves was considered in Ref. [10]. But nowadays, it is commonly accepted that tachyonic fields 
do not propagate with superluminal speeds [11, 12, because it is not possible to localize them on a given hypersurface in the 4-dimensional spacetime, $M_{1,3}$. Thus, the Cauchy data for such fields cannot be specified. Usually, by "tachyonic field" is understood a field that satisfies, e.g., the Klein-Gordon equation with the opposite sign of mass square. But according to the extended relativity [6] - 9] that takes into account not only the subluminal, but also the superluminal Lorentz transformations, the latter fields are not true tachyonic fields. Namely, under a superluminal boost in the $x^{1}$-direction, the coordinates $x^{2}, x^{3}$ and momenta $p^{2}, p^{3}$ become imaginary, and the Klein-Gordon equation transforms into an equation that has the same form as the original equation, in which $x^{0}$ and $x^{1}$ are interchanged. The hypersurface, on which "initial" data can be specified, is now a time-like hypersurface $x^{1}=$ constant. If we keep the definition of velocity as the derivative of the coordinate with respect to $x^{0}$, then the group velocity of the field is given by the derivative of $p^{0}$ with respect to $p^{1}$, and it is greater than the velocity of light. Such a field is the true tachyonic field. It can be obtained from a bradyonic field by a superluminal transformation. The fields that satisfy the Klein-Gordon equation in $M_{1,3}$, with the opposite mass square, cannot be obtained from the bradyonic fields by a superluminal transformation. Therefore, they are not the tachyonic fields of the extended relativity, but are completely different objects that, according to extended relativity, have nothing to do with tachyons. Only the fields that can be obtained from the bradyonic fields by a superluminal transformations can be treated as true tachyonic fields.

The imaginary unit occurring in superluminal transformations (SLT) imply that spacetime is a complex space, $M_{1,3} \times \mathbb{C}$. We can avoid 1 imaginary coordinates, if instead of the $4 D$ complex spacetime we use the real $8 D$ spacetime $M_{4,4}$. The Klein-Gordon equation in such $8 D$ spacetime is ultrahyperbolic, and it describes bradyons and tachyons, depending on the sign of the squared mass. Regardless of whether the field is bradyonic or tachyonic, the Cauchy problem is not well posed, because no space-like or time-like 7D hypersurface exist on which the initial data can be arbitrarily specified. We show explicitly how the initial data can be arbitrarily chosen on lower dimensional space-like or time-like surfaces 2 of dimensionality 4, and point out that this could perhaps be sufficient for a consistent physics of bradyonic and tachyonic fields in $M_{4,4}$.

Another possibility is to consider a space of even higher dimensionality, and take initial data on suitable light-like hypersurfaces in such space. It is well-known [17] that the Cauchy problem for ultrahyperbolic equations is well posed on light-like hypersurfaces. If the latter hypersurfaces are orthogonal to our spacetime $M_{1,3}$, then we arrive at the generalized

\footnotetext{
${ }^{1}$ In some literature (see for example [13-16]), imaginary coordinates, occurring in superluminal transformations, are avoided by considering a 6 -dimensional spacetime, $M_{3,3}$, with 3 time-like and 3 space-like dimensions. Then, under a SLT in the $x^{1}$-direction, the space-like coordinates $x^{2}, x^{3}$ transform into the time-like coordinates $t^{2}, t^{3}$, and vice versa.

${ }^{2}$ Such a $4 D$ time-like surface in $M_{4,4}$, for a fixed extra coordinate, corresponds to the time-like $3 \mathrm{D}$ hyperfurface, $x^{1}=$ constant, mentioned in the previous paragraph.
} 
Stueckelberg equation with a parameter $\tau$ that is invariant under the Lorentz transformations in $M_{1,3}$. A Stueckelberg field $\psi\left(\tau, x^{\mu}\right.$, extra coordinates $), \mu=0,1,2,3$, contains not only bradyons, but also tachyons, because it can be decomposed into components with the 4-momenta $p_{\mu}$ that are not constrained, and so both signs of $p^{\mu} p_{\mu}$ are allowed.

We consider a specific choice for a higher dimensional space, namely the $16 D$ Clifford space, $C$, that has been extensively discussed in the literature [18]-[28]. $C$ is a manifold whose tangent space at any of its points is the Clifford algebra $C l(1,3)$ of the spacetime $M_{1,3}$. Mathematically, $C$ is the space of oriented $r$-volumes, $r=0,1,2,3,4$. Physically, it can be interpreted as a configuration space, associated with extended objects living in $4 D$ spacetime. In this paper we point out, how the Clifford space $C$ leads to the Stueckelberg theory, and thus to a consistent theory of tachyons. We also consider the generalized Dirac equation in $C$, and show that it contains, as particular cases, the tachyonic Dirac equation by Chodos et al. [29], and the bradyonic and tachyonic Dirac equations considered in Ref. [30. Many other important features of Clifford space, such as description of spinors, Kaluza-Klein theories, possible unification of interaction, etc., have already been described in Refs. [18]-28].

\section{The extended relativity and superluminal trans- formations in $M_{1,3}$}

Special relativity can be extended [6]-[9] so to incorporate slower and faster than light particles, the so called bradyons, $B$, and tachyons, $T$, and the superluminal transformations, SLT, that transform $B$ into $T$, and vice versa. In a given reference frame, $S$, a particle is observed as a bradyon, if its velocity is $v<c$, and as a tachyon, if $v>c$. Under a superluminal transformation, the reference frame $S$ transforms into $S^{\prime}$. A particle that has

$v<c$ in $S$, is observed in $S^{\prime}$ to have $v>c$, and vice versa. Whether a particle is bradyon or tachyon depends on the reference frame from which it is observed. Subluminal Lorentz transformations preserve the quadratic form $\mathrm{d} s^{2}=\eta_{\mu \nu} \mathrm{d} x^{\mu} \mathrm{d} x^{\nu}$, so that $\mathrm{d} s^{\prime 2}=\mathrm{d} s^{2}$. On the contrary, superluminal Lorentz transformations change the sign of $\mathrm{d} s^{2}$, so that $\mathrm{d} s^{\prime 2}=-\mathrm{d} s^{2}$. For the Minkowski metric tensor we take $\eta_{\mu \nu}=\operatorname{diag}(1,-1,-1,-1)$.

A superluminal transformation in the $x$-direction, of a position 4 -vector $x^{\mu} \equiv(t, x, y, z)$, $\mu=0,1,2,3$, is [6] -9

$$
t^{\prime}=\frac{t+v x}{\sqrt{v^{2}-1}}, \quad x^{\prime}=\frac{v t+x}{\sqrt{v^{2}-1}}, \quad y^{\prime}=i y, \quad z^{\prime}=i z
$$

It satisfies $\mathrm{d} t^{\prime 2}-\mathrm{d} x^{\prime 2}-\mathrm{d} y^{\prime 2}-\mathrm{d} z^{\prime 2}=-\left(\mathrm{d} t^{2}-\mathrm{d} x^{2}-\mathrm{d} y^{2}-\mathrm{d} z^{2}\right)$. An imaginary unit $i$ occurs in the transformation of $y$ and $z$, otherwise the quadratic form could not change its sign. While transformations among subluminal reference frames can be formulated without reference to complex numbers, this is not the case for transformations between subluminal and superluminal reference frames. 
It has been shown in Refs. [6]-8] that under a superluminal Lorentz transformation, the velocity addition formula remains the same as in the case of a subluminal Lorentz transformations. From (1) we have

$$
\frac{\mathrm{d} x^{\prime}}{\mathrm{d} t^{\prime}} \equiv u^{\prime}=\frac{v+u}{1+v u}, \quad u \equiv \frac{\mathrm{d} x}{\mathrm{~d} t}
$$

which is the same equation that comes out from a subluminal Lorentz transformation. Here $u$ is an object's velocity observed in $S$, and $u^{\prime}$ the velocity observed in $S^{\prime}$, whereas $v$ is the relative velocity between $S$ and $S^{\prime}$. In Eq. (21) one can plug whatever velocities $v \in(-\infty, \infty), u \in(-\infty, \infty)$. As result one obtains a corresponding velocity $u^{\prime}$, that can be either subluminal or superluminal. The case with $v \in(-1,1)$ and $u \in(1,3)$ has been illustrated 3 in Fig. 1 of Ref. [31], where a 2D plot shows that the superluminal velocity $u$, observed in $S$, remains superluminal upon transformation with a subluminal velocity $v$ from a frame $S$ into a frame $S^{\prime}$.

The transformations, analogous to (1), hold for the 4-momentum $p^{\mu}=\left(p^{0}, p^{1}, p^{2}, p^{3}\right) \equiv$ $\left(p_{t}, p_{x}, p_{y}, p_{z}\right)$. The component $p^{0} \equiv p_{t} \equiv E$ is a particle's energy. Under a SLT we have

$$
p^{\prime \mu} p_{\mu}^{\prime}=-p^{\mu} p_{\mu}
$$

If a particle is bradyon in $S^{\prime}$, then it satisfies the mass shell constraint

$$
p^{\prime \mu} p_{\mu}^{\prime}=m^{2}
$$

Under a SLT, this becomes

$$
-p^{\mu} p_{\mu}=m^{2}
$$

Here the proper mass, $m$, is assumed to be invariant under SLT. More explicitly, a bradyon in $S^{\prime}$, with the momentum $\left(p^{\prime 0}, p^{1}, p^{\prime 2}, p^{\prime 3}\right)$, satisfies the mass shell constraint

$$
\left(p^{\prime 0}\right)^{2}-\left(p^{\prime 1}\right)^{2}-\left(p^{\prime 2}\right)^{2}-\left(p^{\prime 3}\right)^{2}=m^{2}
$$

Here $p^{\prime 0}, p^{1}, p^{\prime 2}, p^{\prime 3}$ are all real. The same particle is observed in $S$ as a tachyon with momentum $\left(p^{0}, p^{1}, p^{2}, p^{3}\right)$, satisfying the transformed mass shell constraint

$$
-\left(p^{0}\right)^{2}+\left(p^{1}\right)^{2}+\left(p^{2}\right)^{2}+\left(p^{3}\right)^{2}=m^{2} .
$$

Assuming that $S$ and $S^{\prime}$ are related by the superluminal transformation (11), we have that $p^{0}, p^{1}$ are real, while $p^{2}$, and $p^{3}$ are imaginary. Written in terms of real quantities, $\tilde{p}^{2}=i p^{2}, \tilde{p}^{3}=i p^{3}$, Eq. (17) reads

$$
\left(p^{1}\right)^{2}-\left(p^{0}\right)^{2}-\left(\tilde{p}^{2}\right)^{2}-\left(\tilde{p}^{3}\right)^{2}=m^{2} .
$$

This is just like the usual, bradyonic, mass shell constraint, in which $p^{0}$ and $p^{1}$ are interchanged. This fact has to be taken into account when considering the Klein-Gordon

\footnotetext{
${ }^{3}$ Here the meaning of $u$ and $v$ is interchanged.
} 
equation for tachyons. Now $p^{1}$ has the same role as $p^{0}$ has in the bradyonic case. In other words, the role of energy is now played by $p^{1} \equiv p_{x}$ :

$$
p_{x}= \pm \sqrt{m^{2}+p_{t}^{2}+\tilde{p}_{y}^{2}+\tilde{p}_{z}^{2}}
$$

where $\tilde{p}_{y} \equiv \tilde{p}^{2}, \tilde{p}_{z} \equiv \tilde{p}^{3}$.

The Klein-Gordon equation in the reference frame $S^{\prime}$ is obtained from the constraint (6) , in which we replace momenta with the operators $p_{\mu}^{\prime}=-i \partial_{\mu}^{\prime}$, where $\partial_{\mu}^{\prime} \equiv \partial / \partial x^{\prime \mu}$ :

$$
\left(-\partial_{\mu}^{\prime} \partial^{\prime \mu}-m^{2}\right) \phi^{\prime}\left(x^{\prime}\right)=0
$$

A particular solution is

$$
\phi^{\prime}\left(x^{\prime}\right)=\mathrm{e}^{i p_{\mu}^{\prime} x^{\prime \mu}}=\mathrm{e}^{i\left(p_{t}^{\prime} t^{\prime}-p_{x}^{\prime} x^{\prime}-p_{y}^{\prime} y^{\prime}-p_{z}^{\prime} z^{\prime}\right)} .
$$

Under a SLT, we have

$$
\phi^{\prime}\left(x^{\prime}\right)=\phi(x)=\mathrm{e}^{-i p_{\mu} x^{\mu}}=\mathrm{e}^{-i\left(p_{t} t-p_{x} x-p_{y} y-p_{z} z\right)}=\mathrm{e}^{-i\left(p_{t} t-p_{x} x+\tilde{p}_{y} \tilde{y}+\tilde{p}_{z} \tilde{z}\right)}
$$

In the last step we have taken into account that $p_{y}, p_{z}, y, z$ are imaginary, and expressed them in terms of the real quantities $\tilde{p}_{y}=i p_{y}, \tilde{p}_{z}=i p_{z}, \tilde{y}=i y, \tilde{z}=i z$.

In the reference frame $S$ we have the Klein-Gordon equation, obtained from the constraint (7), which is equivalent to the constraint (8):

$$
\begin{aligned}
\left(\partial_{\mu} \partial^{\mu}-m^{2}\right) \phi(x) & =\left(\frac{\partial^{2}}{\partial t^{2}}-\frac{\partial^{2}}{\partial x^{2}}-\frac{\partial^{2}}{\partial y^{2}}-\frac{\partial^{2}}{\partial z^{2}}-m^{2}\right) \phi(t, x, y, z) \\
& =\left(\frac{\partial^{2}}{\partial t^{2}}-\frac{\partial^{2}}{\partial x^{2}}+\frac{\partial^{2}}{\partial \tilde{y}^{2}}+\frac{\partial^{2}}{\partial \tilde{z}^{2}}-m^{2}\right) \phi(t, x, \tilde{y}, \tilde{z})=0 .
\end{aligned}
$$

From the mathematical point of view, this is just like the usual Klein-Gordon equation, with $t$ and $x$ interchanged. A particular solution of the latter equation is (12).

A general solution is

$$
\phi=\int \mathrm{d} p_{t} \mathrm{~d} p_{x} \mathrm{~d} \tilde{p}_{y} \mathrm{~d} \tilde{p}_{z} c\left(p_{t}, p_{x}, \tilde{p}_{y}, \tilde{p}_{z}\right) \mathrm{e}^{i\left(p_{x} x-p_{t} t-\tilde{p}_{y} \tilde{y}-\tilde{p}_{z} \tilde{z}\right)} \delta\left(p_{x}^{2}-p_{t}^{2}-\tilde{p}_{y}^{2}-\tilde{p}_{z}^{2}-m^{2}\right),
$$

where $c\left(p_{t}, p_{x}, \tilde{p}_{y}, \tilde{p}_{z}\right)$ is a function of its arguments, restricted to the mass shell. Introducing $\omega_{x}=\left|\sqrt{m^{2}+p_{t}^{2}+\tilde{p}_{y}^{2}+\tilde{p}_{z}^{2}}\right|$, and integrating over $p_{x}= \pm \omega_{x}$, we obtain

$$
\begin{aligned}
\phi=\int \mathrm{d} p_{t} \mathrm{~d} \tilde{p}_{y} \mathrm{~d} \tilde{p}_{z} \frac{1}{2 \pi \omega_{x}}\left[\mathrm{e}^{i\left(\omega_{x} x-p_{t} t-\tilde{p}_{y} \tilde{y}-\tilde{p}_{z} \tilde{z}\right)} c\left(\omega_{x}, p_{t}, \tilde{p}_{y}, \tilde{p}_{z}\right)\right. \\
\\
\left.+\mathrm{e}^{i\left(-\omega_{x} x-p_{t} t-\tilde{p}_{y} \tilde{y}-\tilde{p}_{z} \tilde{z}\right)} c\left(-\omega_{x}, p_{t}, \tilde{p}_{y}, \tilde{p}_{z}\right)\right]
\end{aligned}
$$

The "initial" data can be given on a surface $x=x_{0}=$ constant. The latter surface is timelike, and is spanned by coordinates $(t, \tilde{y}, \tilde{z})$. Specifying "initial" data on a hypersurface 
$x=x_{0}$, one can calculate the field $\phi$ at other hypersurfaces, with different values of $x$. That a Cauchy surface for space-like states must be time-like, was discussed in Ref. [11, 32, but without employing superluminal transformations, and in Ref. 33, 34, within the framework of 2-dimensional spacetime.

Let us now investigate how does move the wave packet that satisfied the Klein-Gordon equation. For this purpose it is convenient to consider the nonrelativistic approximation to the Klein-Gordon equation. Writing a solution of Eq. (10) as $\phi^{\prime}=\mathrm{e}^{-i m t^{\prime}} \psi^{\prime}\left(t^{\prime}, \mathbf{x}^{\prime}\right)$, and substituting it into Eq. (10), we obtain [35]

$$
-\frac{1}{2 m} \frac{\partial^{2} \psi^{\prime}}{\partial t^{\prime 2}}+i \frac{\partial \psi^{\prime}}{\partial t^{\prime}}=-\frac{1}{2 m} \nabla^{\prime 2} \psi^{\prime}
$$

In the nonrelativistic limit we can neglect the first term, and we obtain the Schrödinger equation

$$
i \frac{\partial \psi^{\prime}}{\partial t^{\prime}}=-\frac{1}{2 m} \nabla^{\prime 2} \psi^{\prime}
$$

A solution to the latter equation is a Gaussian wave packet, whose square is

$$
\left|\psi^{\prime}\right|^{2} \propto \exp \left[-\frac{\left(\mathbf{x}^{\prime}-\frac{\mathbf{p}_{0}^{\prime}}{m} t^{\prime}\right)^{2}}{\sigma^{\prime}\left(t^{\prime}\right)}\right]
$$

where $\sigma^{\prime}\left(t^{\prime}\right)=\sigma_{0}^{\prime}+t^{\prime 2} /\left(m^{2} \sigma_{0}^{\prime}\right)$. The probability density is maximal on the classical trajectory $\mathbf{x}^{\prime}=\left(\mathbf{p}_{0}^{\prime} / m\right) t^{\prime}$ with velocity $\mathbf{v}^{\prime}=\mathbf{p}_{0}^{\prime} / m,\left|\mathbf{v}^{\prime}\right|<c=1$. We have verified that the Klein-Gordon Eq. (10) in the reference frame $S^{\prime}$ describes a field, propagating with a slower than light velocity $\mathbf{v}^{\prime}$ (Fig. 1a).

The same procedure can be repeated for the Klein-Gordon equation (13) that holds in the reference frame $S$, and in which $t$ and $x$ are interchanged. Writing $\phi=\mathrm{e}^{-i m x} \psi$, and substituting this into Eq. (13), we obtain

$$
-\frac{1}{2 m} \frac{\partial^{2} \psi}{\partial x^{2}}+i \frac{\partial \psi}{\partial x}=-\frac{1}{2 m}\left(\frac{\partial^{2}}{\partial t^{2}}+\frac{\partial^{2}}{\partial \tilde{y}^{2}}+\frac{\partial^{2}}{\partial \tilde{z}^{2}}\right) \psi
$$

In the approximation, in which the first term can be neglected, we have

$$
i \frac{\partial \psi}{\partial x}=-\frac{1}{2 m}\left(\frac{\partial^{2}}{\partial t^{2}}+\frac{\partial^{2}}{\partial \tilde{y}^{2}}+\frac{\partial^{2}}{\partial \tilde{z}^{2}}\right) \psi
$$

Such equation, but with the $x$ and $t$ variables only, was considered by Vyšín [33, 34]. A solution of the Schrödinger-like equation (20) is the wave packet whose square is

$$
|\psi|^{2} \propto \exp \left[-\frac{\left(t-\frac{p_{t 0}}{m} x\right)^{2}}{\sigma(x)}-\frac{\left(\tilde{y}-\frac{\tilde{p}_{y 0}}{m} x\right)^{2}}{\sigma(x)}-\frac{\left(\tilde{z}-\frac{p_{\tilde{z} 0}}{m} x\right)^{2}}{\sigma(x)}\right],
$$

where $\sigma(x)=\sigma_{0}+x^{2} /\left(m^{2} \sigma_{0}\right)$. The probability density is now picked on the classical trajectory, $t=\frac{p_{t 0}}{m} x, \tilde{y}-\frac{\tilde{p}_{y 0}}{m}, \tilde{z}-\frac{\tilde{p}_{z 0}}{m}$, where $p_{t 0} / m=\mathrm{d} t / \mathrm{d} x, \tilde{p}_{y 0} / m=\mathrm{d} \tilde{y} / \mathrm{d} x$, and $\tilde{p}_{z 0} / m=$ 
$\mathrm{d} \tilde{z} / \mathrm{d} x,\left|\sqrt{(\mathrm{d} t / \mathrm{d} x)^{2}+(\mathrm{d} \tilde{y} / \mathrm{d} x)^{2}+(\mathrm{d} \tilde{z} / \mathrm{d} x)^{2}}\right|<c=1$. If we keep on considering $t$ as a time coordinate, then the velocity of the wave packet (21) in the $x$-direction should be defined according to $v_{x}=\mathrm{d} x / \mathrm{d} t=m / p_{t 0}$, where $\left|v_{x}\right|>1$. The reciprocity between time and space for bradyons and tachyons was noticed long time ago [9, 36], and localization of tachyons on the basis of such reciprocity was considered by Višyn [33, 34], [11, and others [37]-39].

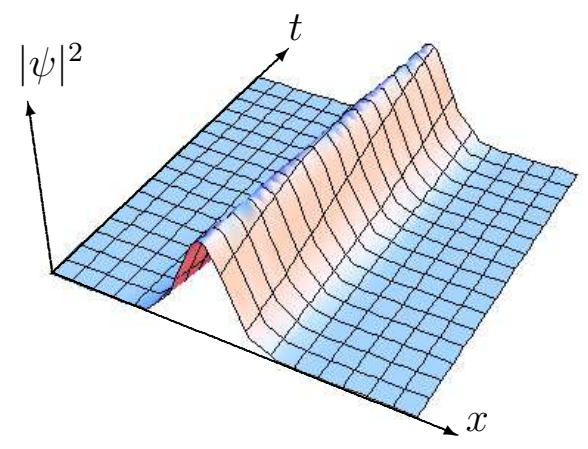

a)

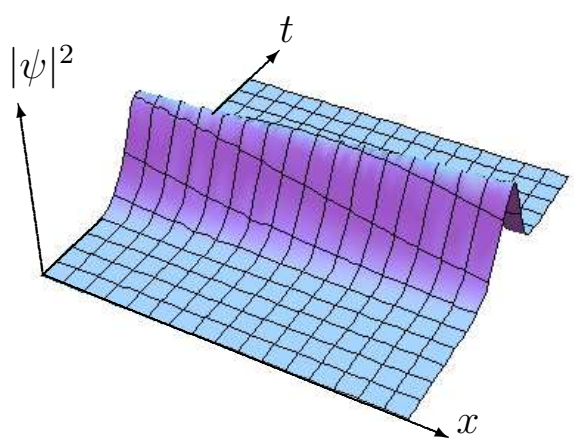

b)

Figure 1: Examples of bradyonic (a) and tachyonic (b) wave packets.

We see that the wave packet (21) propagates with the superluminal velocity (Fig. 1b). Because the subluminal wave packet (18) can transmit a signal, so can the wave packet (21), which is nothing but a superluminal transform of (18).

We illustrated the situation by considering the nonrelativistic wave packet. In the relativistic case, we can use the dispersion relation (9). We find that

$$
\frac{\mathrm{d} p_{x}}{\mathrm{~d} p_{t}}=\frac{\mathrm{d} t}{\mathrm{~d} x}=\frac{p_{t}}{\sqrt{p_{t}^{2}+\tilde{p}_{y}^{2}+\tilde{p}_{z}^{2}+m^{2}}}, \quad\left|\frac{\mathrm{d} t}{\mathrm{~d} x}\right|<c=1
$$

gives the reciprocal group velocity of the tachyonic field in the $x$-direction. It follows that $\mathrm{d} x / \mathrm{d} t>1$. For simplicity, we now omit the subscript ' 0 ', denoting the center of the wave packet. That a tachyonic field propagates with a superluminal speed directly follows from the relativistic dispersion relation $p_{t}=\sqrt{p_{x}^{2}-\tilde{p}_{y}^{2}-\tilde{p}_{z}^{2}-m^{2}}$ that comes from Eq. (8), and from which we obtain the faster-than-light group velocity

$$
\frac{\mathrm{d} p_{t}}{\mathrm{~d} p_{x}}=\frac{\mathrm{d} x}{\mathrm{~d} t}=\frac{p_{x}}{\sqrt{p_{x}^{2}-\tilde{p}_{y}^{2}-\tilde{p}_{z}^{2}-m^{2}}}, \quad\left|\frac{\mathrm{d} x}{\mathrm{~d} t}\right|>c=1
$$

The nonrelativistic (i.e., very fast moving) tachyon wave packet is localized in time, and also in space. From Fig. 1b and Eq. (21) we see that the localization width in space is wide in comparison with that in time. This is a non relativistic approximation. A relativistic tachyon can be localized only in time, but not in space. The fact that tachyons are infinitely extended in space, was taken as an argument against the possibility that they 
can transmit information. However, as already observed by Vyšín [33, 34], it is important that tachyons form sharp pulses in time. A sequence of such pulses, localized in time, can encode information. If there is an interaction between tachyons and a bradyonic detector, then a bradyonic observer $B$ would be able to observe that sequence of pulses, and interpret it as the information, emitted by another observer $A$. This is illustrated in Fig. 2.

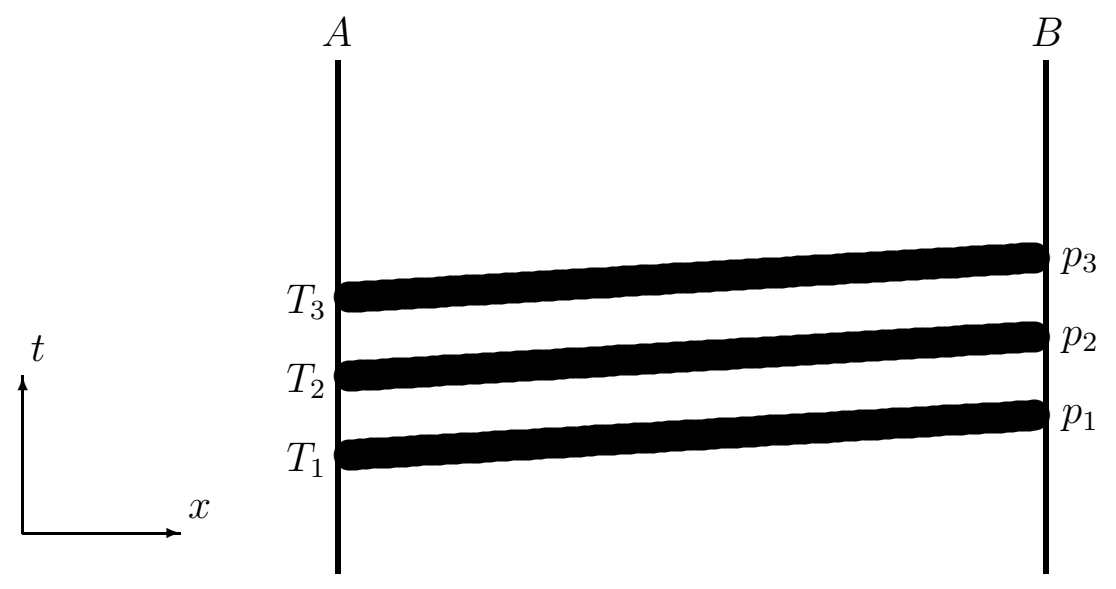

Figure 2: Though the tachyons wave packets are not localized in the $x$-direction, having no sharp peaks and leading edges along the $x$-direction, they can nevertheless transmit information from $A$ to be $B$, because they are localized in time. Namely, if tachyons can interact with bradyons, then the observer $A$ can send a message encoded in a sequence of emitted tachyons, $T_{1}, T_{2}, T_{3}, \ldots$, that are detected by the observer $B$ as a sequence of pulses, $p_{1}, p_{2}, p_{3}, \ldots$, at a fixed spatial position. $B$ can unambiguously interpret such sequence as a message, if both observers had already agreed about the code.

The solution (14) is the superluminal transform of the general solution of the KleinGordon equation (10) for a bradyonic field in $S^{\prime}$. Under a SLT, the bradyonic KleinGordon equation transforms into the tachyonic Klein-Gordon equation. But according to the principle of relativity, the laws of motion, encrypted in the equations of motion, should remain unchanged under all transformations that bring one dynamically possible solution into another dynamically possible solution. According to our assumption, SLT are such transformations. Therefore, the equations of motion should remain invariant under SLT. Since Eq. (10) is not invariant, it means that it is not a complete equation, but a part of a more general equation. In the following we will consider a more general theory which the Klein-Gordon equation (13) is embedded in. First, we will discuss a generalization of the classical theory of the relativistic point particle, and then its quantization. 


\section{Complex coordinates and momenta, and the real spacetime $M_{4,4}$}

A superluminal boost (11) contains imaginary quantities. After performing, successively, a superluminal boost, rotations and a subluminal boost, we will end up with complex spacetime coordinates and momenta:

$$
X^{\mu}=x^{\mu}+i \tilde{x}^{\mu}, \quad P^{\mu}=p^{\mu}+i \tilde{p}^{\mu},
$$

with

$$
\begin{gathered}
X^{\mu} X_{\mu}=X^{\mu} X^{\nu} \eta_{\mu \nu}=x^{\mu} x_{\mu}-\tilde{x}^{\mu} \tilde{x}_{\mu}+2 i x^{\mu} \tilde{x}_{\mu}, \\
P^{\mu} P_{\mu}=P^{\mu} P^{\nu} \eta_{\mu \nu}=p^{\mu} p_{\mu}-\tilde{p}^{\mu} \tilde{p}_{\mu}+2 i p^{\mu} \tilde{p}_{\mu},
\end{gathered}
$$

Subluminal Lorentz transformations preserve $X^{\mu} X_{\mu}$, so they preserve $x^{\mu} x_{\mu}-\tilde{x}^{\mu} \tilde{x}_{\mu}$ and $x^{\mu} \tilde{x}_{\mu}$. Superluminal Lorentz transformations change the sign of $X^{\mu} X_{\mu}$. A superluminal boost in the $x$-direction reads

$$
T^{\prime}=\frac{T+v X}{\sqrt{v^{2}-1}}, \quad X^{\prime}=\frac{v T+X}{\sqrt{v^{2}-1}}, \quad Y^{\prime}=i Y, \quad Z^{\prime}=i Z,
$$

where $v$ is real. For the real and imaginary components, we have

$$
\begin{gathered}
t^{\prime}=\frac{t+v x}{\sqrt{v^{2}-1}}, \quad x^{\prime}=\frac{v t+x}{\sqrt{v^{2}-1}}, \quad y^{\prime}=-\tilde{y}, \quad z^{\prime}=-\tilde{z} \\
\tilde{t}^{\prime}=\frac{\tilde{t}+v \tilde{x}}{v^{2}-1}, \quad \tilde{x}^{\prime}=\frac{v \tilde{t}+\tilde{x}}{v^{2}-1}, \quad \tilde{y}^{\prime}=y, \quad \tilde{z}^{\prime}=z
\end{gathered}
$$

Analogous transformations hold for the momentum $P^{\mu}$. In this setup with complex coordinates and momenta, the multiplication by $i$ in the SLT (27) means the interchange of the real and imaginary components.

If in the reference frame $S^{\prime}$, the imaginary components are zero, $\tilde{x}^{\prime \mu}=0, \tilde{p}^{\prime \mu}=0$, then in $S$, according to Eqs. (28), (29), we have

$$
\begin{gathered}
y=0, \quad z=0, \quad \tilde{y}=-y^{\prime}, \quad \tilde{z}=-z^{\prime} \\
p^{2}=0, \quad p^{3}=0, \quad \tilde{p}^{2}=-p^{2}, \quad \tilde{p}^{3}=-p^{\prime 3}
\end{gathered}
$$

The quadratic forms read

$$
\begin{gathered}
X^{\prime \mu} X_{\mu}^{\prime}=x^{\prime \mu} x_{\mu}^{\prime}=t^{\prime 2}-x^{\prime 2}-y^{\prime 2}-z^{\prime 2} \\
=-t^{2}+x^{2}-y^{\prime 2}-z^{\prime 2}=-t^{2}+x^{2}-\tilde{y}^{2}-\tilde{z}^{2} \\
P^{\prime \mu} P_{\mu}^{\prime}=p^{\prime \mu} p_{\mu}^{\prime}=\left(p^{\prime 0}\right)^{2}-\left(p^{\prime 1}\right)^{2}-\left(p^{\prime 2}\right)^{2}-\left(p^{\prime 3}\right)^{2} \\
=-\left(p^{0}\right)^{2}+\left(p^{1}\right)^{2}-\left(p^{\prime 2}\right)^{2}-\left(p^{\prime 3}\right)^{2}=-\left(p^{0}\right)^{2}+\left(p^{1}\right)^{2}-\left(\tilde{p}^{2}\right)^{2}-\left(\tilde{p}^{3}\right)^{2}
\end{gathered}
$$


This is precisely the quadratic form of Eq. (8) that we arrived at in Sec. 2. The derivation through Eqs. (11) - (15) belongs to a special case of a more general theory in which coordinates and momenta are complex.

Let the momentum constraint in the reference frame $S^{\prime}$ be

$$
\operatorname{Re} P^{\prime \mu} P_{\mu}^{\prime}+M^{2}=0
$$

Assuming real $M^{2}$, we thus have

$$
p^{\prime \mu} p_{\mu}^{\prime}-\tilde{p}^{\prime \mu} \tilde{p}_{\mu}^{\prime}+M^{2}=0
$$

In a reference frame $S$, related to $S^{\prime}$ by the SLT (28),(29), we have

$$
-\left(p^{0}\right)^{2}+\left(p^{1}\right)^{2}+\left(p^{2}\right)^{2}+\left(p^{3}\right)^{2}+\left(\tilde{p}^{0}\right)^{2}-\left(\tilde{p}^{1}\right)^{2}-\left(\tilde{p}^{2}\right)^{2}-\left(\tilde{p}^{3}\right)^{2}+M^{2}=0
$$

i.e.,

$$
-p^{\mu} p_{\mu}+\tilde{p}^{\mu} \tilde{p}_{\mu}+M^{2}=0
$$

Here, again $M^{2}$ is assumed to be invariant under SLT. It may be positive, or negative:

a) If $M^{2}>0$, then the particle is observed as a tachyon in $S^{\prime}$, and as a bradyon in $S$.

b) If $M^{2}<0$, then the particle is observed as a bradyon in $S^{\prime}$, and as a tachyon in $S$.

Instead of the 4-dimensional complex spacetime $M_{1,3} \times \mathbb{C}$, we can consider the 8dimensional real space $M_{4,4}$ with signature $(4,4)$.

Upon quantization, the constraint (37) becomes the Klein-Gordon equation:

$$
\left(\partial^{\mu} \partial_{\mu}-\tilde{\partial}^{\mu} \tilde{\partial}_{\mu}+M^{2}\right) \phi\left(x^{\mu}, \tilde{x}^{\mu}\right)=0
$$

where $\tilde{\partial}^{\mu} \tilde{\partial}_{\mu}=\tilde{\partial}^{\mu} \tilde{\partial}^{\nu} \eta_{\mu \nu}$, and $\tilde{\partial}_{\mu} \equiv \partial / \partial \tilde{x}^{\mu}$.

A general solution of this equation is

$$
\phi=\int \mathrm{d}^{4} p \mathrm{~d}^{4} \tilde{p} c(p, \tilde{p}) \mathrm{e}^{i\left(p_{\mu} x^{\mu}-\tilde{p}_{\mu} \tilde{x}^{\mu}\right)} \delta\left(p^{\mu} p_{\mu}-\tilde{p}^{\mu} \tilde{p}_{\mu}-M^{2}\right)
$$

Instead of one, we have now four time-like and four space-like coordinates and momenta:

$$
\begin{array}{lll}
\left(x^{0}, \tilde{x}^{1}, \tilde{x}^{2}, \tilde{x}^{3}\right) ; & \left(p^{0}, \tilde{p}^{1}, \tilde{p}^{2}, \tilde{p}^{3}\right) & \text { time-like } \\
\left(x^{1}, x^{2}, x^{3}, \tilde{x}^{0}\right) ; & \left(p^{1}, p^{2}, p^{3}, \tilde{p}^{0}\right) & \text { space-like }
\end{array}
$$

In the case of the bradyonic field, $M^{2}>0$, we can proceed as follows. We choose the time-like component $p^{0}$ and integrate it out in Eq. (39). Denoting $\mathbf{x} \equiv\left(x^{1}, x^{2}, x^{3}\right)$, etc., we obtain:

$$
\begin{aligned}
\phi\left(x^{\mu}, \tilde{x}^{\mu}\right)=\int \mathrm{d}^{3} \mathbf{p} \mathrm{d} \tilde{p}^{0} \mathrm{~d}^{3} \tilde{\mathbf{p}} \frac{1}{2 \omega}\left[\mathrm{e}^{i\left(\omega x^{0}-\mathbf{p x}-\tilde{p}_{0} \tilde{x}^{0}+\tilde{\mathbf{p}} \tilde{\mathbf{x}}\right)} c\left(\omega, \mathbf{p}, \tilde{p}^{0}, \tilde{\mathbf{p}}\right)\right. \\
\left.+\mathrm{e}^{i\left(-\omega x^{0}-\mathbf{p x}-\tilde{p}_{0} \tilde{x}^{0}+\tilde{\mathbf{p}} \tilde{\mathbf{x}}\right)} c\left(-\omega, \mathbf{p}, \tilde{p}^{0}, \tilde{\mathbf{p}}\right)\right]
\end{aligned}
$$


where

$$
p^{0}= \pm \omega, \quad \omega=\left|\sqrt{\left(\tilde{p}^{0}\right)^{2}+\mathbf{p}^{2}-\tilde{\mathbf{p}}^{2}+M^{2}}\right|
$$

At the (7-dimensional) hypersurface $x^{0}=0$, the initial data $\left.\phi\right|_{x^{0}=0}$ and $\left.\left(\mathrm{d} \phi / \mathrm{d} x^{0}\right)\right|_{x^{0}=0}$ cannot be arbitrarily chosen. Because the momenta, assumed to be real, are constrained by Eq. (41), the expression (40) does not give arbitrary $\left.\phi\right|_{x^{0}=0}$. Similarly for the $x^{0}$-derivative of $\phi$. The Cauchy problem is thus not well posed [40].

This is the notorious property of ultrahyperbolic partial differential equations that involve more than one time-like dimension. Usually, this is considered as a problem, and it is concluded that ultrahyperbolic equations, such as (38), are not suitable for physics. I think that the requirement that initial data should be given arbitrarily on a hypersurface (which in our case is a 7-surface), is an unnecessary restriction on which equations are admissible in physics. We will discuss this in more detail later. In the case of Eq. (38), the initial data can be freely specified on the 4 -surface, spanned by coordinates $\left(x^{1}, x^{2}, x^{3}, \tilde{x}^{0}\right)$. This follows immediately from Eq. (40). Putting $x^{0}=0, \tilde{\mathbf{x}}=\mathbf{0}$, we have

$$
\begin{aligned}
\phi\left(0, \mathbf{x}, \tilde{x}^{0}, \mathbf{0}\right) & =\int \mathrm{d}^{3} \mathbf{p} \mathrm{d} \tilde{p}^{0} \mathrm{~d}^{3} \tilde{\mathbf{p}} \frac{1}{2 \omega} \mathrm{e}^{i\left(-\mathbf{p x}-\tilde{p}_{0} \tilde{x}^{0}\right)}\left[c\left(\omega, \mathbf{p}, \tilde{p}^{0}, \tilde{\mathbf{p}}\right)+c\left(-\omega, \mathbf{p}, \tilde{p}^{0}, \tilde{\mathbf{p}}\right)\right] \\
= & \int_{-\infty}^{\infty} \mathrm{d}^{3} \mathbf{p} \mathrm{d} \tilde{p}^{0} A\left(\mathbf{p}, \tilde{p}^{0}\right) \mathrm{e}^{-i\left(\mathbf{p} \mathbf{x}+\tilde{p}_{0} \tilde{x}^{0}\right)}
\end{aligned}
$$

where

$$
A\left(\mathbf{p}, \tilde{p}_{0}\right)=\int_{\tilde{\mathbf{p}}^{2} \leq M^{2}+\left(\tilde{p}^{0}\right)^{2}+\mathbf{p}^{2}} \mathrm{~d}^{3} \tilde{\mathbf{p}} \frac{1}{2 \omega}\left[c\left(\omega, \mathbf{p}, \tilde{p}^{0}, \tilde{\mathbf{p}}\right)+c\left(-\omega, \mathbf{p}, \tilde{p}^{0}, \tilde{\mathbf{p}}\right)\right]
$$

The initial data for the derivatives of the field with respect to the time-like coordinates $x^{0}, \tilde{\mathbf{x}}$, are

$$
\begin{aligned}
& \frac{\partial \phi\left(0, \mathbf{x}, \tilde{x}^{0}, \mathbf{0}\right)}{\partial x^{0}}=\int \mathrm{d}^{3} \mathbf{p} \mathrm{d} \tilde{p}^{0} B\left(\mathbf{p}, \tilde{p}^{0}\right) \mathrm{e}^{-i\left(\mathbf{p x}+\tilde{p}_{0} \tilde{x}^{0}\right)} \\
& \frac{\partial \phi\left(0, \mathbf{x}, \tilde{x}^{0}, \mathbf{0}\right)}{\partial \tilde{\mathbf{x}}}=\int \mathrm{d}^{3} \mathbf{p} \mathrm{d} \tilde{p}^{0} \mathbf{C}\left(\mathbf{p}, \tilde{p}^{0}\right) \mathrm{e}^{-i\left(\mathbf{p} \mathbf{x}+\tilde{p}_{0} \tilde{x}^{0}\right)},
\end{aligned}
$$

where

$$
\begin{gathered}
B\left(\mathbf{p}, \tilde{p}^{0}\right)=\int_{\tilde{\mathbf{p}}^{2} \leq M^{2}+\left(\tilde{p}^{0}\right)^{2}+\mathbf{p}^{2}} \mathrm{~d}^{3} \tilde{\mathbf{p}} \frac{1}{2}\left[c\left(\omega, \mathbf{p}, \tilde{p}^{0}, \tilde{\mathbf{p}}\right)+c\left(-\omega, \mathbf{p}, \tilde{p}^{0}, \tilde{\mathbf{p}}\right)\right], \\
\mathbf{C}\left(\mathbf{p}, \tilde{p}^{0}\right)=\int_{\tilde{\mathbf{p}}^{2} \leq M^{2}+\left(\tilde{p}^{0}\right)^{2}+\mathbf{p}^{2}} \mathrm{~d}^{3} \tilde{\mathbf{p}} \frac{\tilde{\mathbf{p}}}{2 \omega}\left[c\left(\omega, \mathbf{p}, \tilde{p}^{0}, \tilde{\mathbf{p}}\right)+c\left(-\omega, \mathbf{p}, \tilde{p}^{0}, \tilde{\mathbf{p}}\right)\right],
\end{gathered}
$$

Here $\mathbf{p}, \tilde{p}^{0}$ are not restricted, but may have values between $-\infty, \infty$, and $A\left(\mathbf{p}, \tilde{p}_{0}\right), B\left(\mathbf{p}, \tilde{p}_{0}\right)$, $\mathbf{C}\left(\mathbf{p}, \tilde{p}_{0}\right)$ are arbitrary 4 functions of $\mathbf{p}, \tilde{p}^{0}$. Therefore, $\phi\left(0, \mathbf{x}, \tilde{x}^{0}, \mathbf{0}\right), \partial \phi\left(0, \mathbf{x}, \tilde{x}^{0}, \mathbf{0}\right) / \partial x^{0}$, $\partial \phi\left(0, \mathbf{x}, \tilde{x}^{0}, \mathbf{0}\right) / \partial \tilde{\mathbf{x}}$ are arbitrary functions of the space-like coordinates $\left(\mathbf{x}, \tilde{x}^{0}\right)$.

\footnotetext{
${ }^{4}$ The functions are arbitrary within the limitation of field theory and quantum mechanics.
} 
From Eq. (43), (46), (47) we see that the expansion coefficients $A\left(\mathbf{p}, \tilde{p}_{0}\right), B\left(\mathbf{p}, \tilde{p}_{0}\right)$, $\mathbf{C}\left(\mathbf{p}, \tilde{p}_{0}\right)$ are the integrals over the extra time-like momenta $\tilde{\mathbf{p}} \equiv\left(\tilde{p}^{1}, \tilde{p}^{2}, \tilde{p}^{3}\right)$, and that they partially determine the expansion coefficients $c\left(\omega, \mathbf{p}, \tilde{p}^{0}, \tilde{\mathbf{p}}\right)$ and $c\left(-\omega, \mathbf{p}, \tilde{p}^{0}, \tilde{\mathbf{p}}\right)$, and thus give us some, although not complete, information on the behavior of the field outside the 4-surface on which the initial data are given. Thus, given the initial data (42), (44), (45) on a space-like 4 -surface, the field $\phi\left(x^{\mu}, \tilde{x}^{\mu}\right)$ is not uniquely determined. This is in conflict with the requirement that a physical theory should be deterministic. But is it indeed necessary for a physical theory to be deterministic? What about quantum theory? In quantum theory, behavior of a field is not deterministic. Perhaps ultrahyperbolic field equations point towards the roots of quantum (field) theory. Since classical field theory, based on an ultrahyperbolic equation, is not deterministic, it should be supplemented (or replaced) by a more general theory which takes into account the indeterminism. Such theory is quantum mechanics. We leave this intriguing point for future investigations.

In particular, if

$$
\begin{aligned}
c\left(\omega, \mathbf{p}, \tilde{p}^{0}, \tilde{\mathbf{p}}\right) & =\delta^{3}\left(\tilde{\mathbf{p}}-\tilde{\mathbf{p}}_{\mathrm{c}}\right) a\left(\omega, \mathbf{p}, \tilde{p}^{0}\right) \\
c\left(-\omega, \mathbf{p}, \tilde{p}^{0}, \tilde{\mathbf{p}}\right) & =\delta^{3}\left(\tilde{\mathbf{p}}-\tilde{\mathbf{p}}_{\mathrm{c}}\right) a\left(-\omega, \mathbf{p}, \tilde{p}^{0}\right),
\end{aligned}
$$

where $\tilde{\mathbf{p}}_{\mathrm{c}}^{2} \leq M^{2}$, then

$$
\begin{gathered}
A\left(\mathbf{p}, \tilde{p}_{0}\right)=\frac{1}{2 \omega_{\tilde{\mathbf{p}}_{c}}}\left[a\left(\omega_{\tilde{\mathbf{p}}_{c}}, \mathbf{p}, \tilde{p}^{0}\right)+a\left(-\omega_{\tilde{\mathbf{p}}_{c}}, \mathbf{p}, \tilde{p}^{0}\right)\right] \\
B\left(\mathbf{p}, \tilde{p}_{0}\right)=\frac{1}{2}\left[a\left(\omega_{\tilde{\mathbf{p}}_{c}}, \mathbf{p}, \tilde{p}^{0}\right)+a\left(-\omega_{\tilde{\mathbf{p}}_{c}}, \mathbf{p}, \tilde{p}^{0}\right)\right] \\
\mathbf{C}\left(\mathbf{p}, \tilde{p}_{0}\right)=\frac{\tilde{\mathbf{p}}_{c}}{2 \omega_{\tilde{\mathbf{p}}_{c}}}\left[a\left(\omega_{\tilde{\mathbf{p}}_{c}}, \mathbf{p}, \tilde{p}^{0}\right)+a\left(-\omega_{\tilde{\mathbf{p}}_{c}}, \mathbf{p}, \tilde{p}^{0}\right)\right],
\end{gathered}
$$

where $\omega_{\tilde{\mathbf{p}}_{c}} \equiv\left|\sqrt{\left(\tilde{p}^{0}\right)^{2}+\mathbf{p}^{2}-\tilde{\mathbf{p}}_{c}^{2}+M^{2}}\right|$. The form (48) of the expansion coefficients means that the extra time-like momenta have a fixed definite value $\tilde{\mathbf{p}}=\tilde{\mathbf{p}}_{\mathrm{c}}$. Then we recover the usual hyperbolic case of one time-like coordinate $x^{0}$, and momentum $p^{0}$. In our theory, we also have an additional extra space like coordinate, $\tilde{x}^{0}$, and an additional component of momentum, $\tilde{p}^{0}$.

If the expansion coefficients are given by Eq. (48), (49), and $M^{2}-\tilde{\mathbf{p}}_{c}^{2}>0$, then the remaining components of momentum, $\mathbf{p}, \tilde{p}^{0}$ all have values between $-\infty$ and $+\infty$. The wave packet (40) has then the form

$\phi\left(x^{\mu}, \tilde{x}^{\mu}\right)=\mathrm{e}^{i \tilde{\mathbf{p}}_{c} \tilde{\mathbf{x}}} \int \mathrm{d}^{3} \mathbf{p} \mathrm{d} \tilde{p}^{0} \frac{1}{2 \omega_{\tilde{\mathbf{p}}_{c}}}\left[\mathrm{e}^{i \omega_{\tilde{\mathbf{p}}_{c}} x^{0}} a\left(\omega_{\tilde{\mathbf{p}}_{c}}, \tilde{p}^{0}, \mathbf{p}\right)+\mathrm{e}^{-i \omega_{\tilde{\mathbf{p}}_{c}} x^{0}} a\left(-\omega_{\tilde{\mathbf{p}}_{c}}, \tilde{p}^{0}, \mathbf{p}\right)\right] \mathrm{e}^{-i\left(\mathbf{p} \mathbf{x}+\tilde{p}_{0} \tilde{x}^{0}\right)}$

It can be arbitrarily localized on the surface $x^{0}=0, \tilde{\mathbf{x}}=0$ that is spanned by coordinates $\left(x^{1}, x^{2}, x^{3}, \tilde{x}^{0}\right)$, but it is delocalized in the time-like directions $\left(x^{0}, \tilde{x}^{1}, \tilde{x}^{2}, \tilde{x}^{3}\right)$ (Fig. 3a).

In the case of the tachyonic field, $M^{2}<0$, the Klein-Gordon equation reads

$$
\left(\partial^{\mu} \partial_{\mu}-\tilde{\partial}^{\mu} \tilde{\partial}_{\mu}-\tilde{M}^{2}\right) \tilde{\phi}\left(x^{\mu}, \tilde{x}^{\mu}\right)=0,
$$



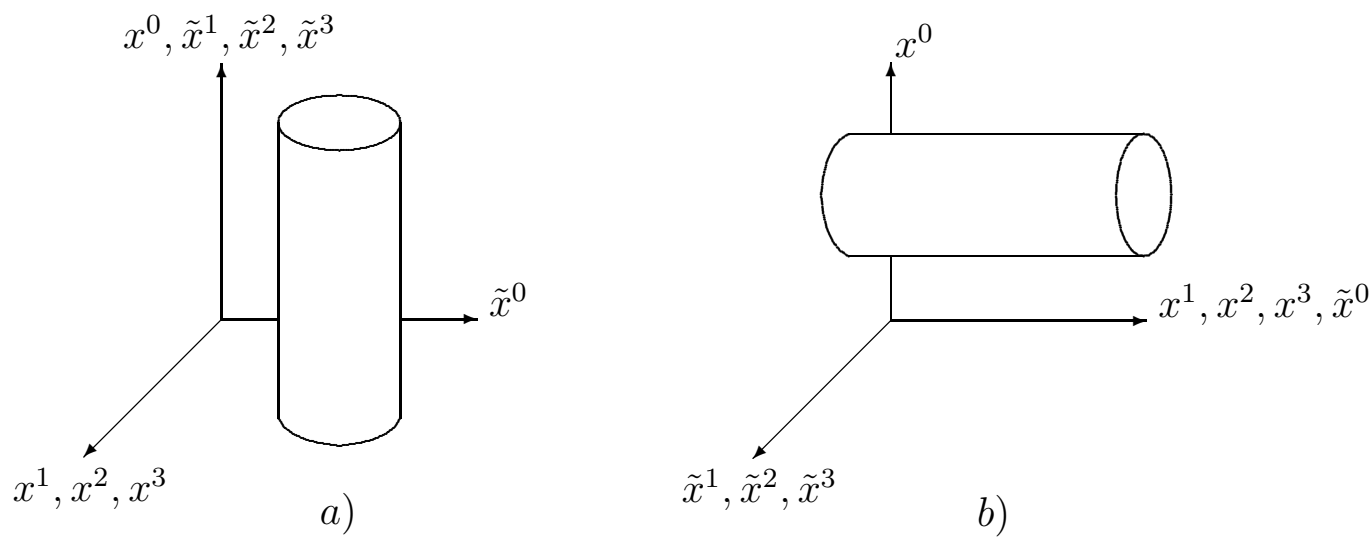

Figure 3: a) A bradyonic field can be localized within a region of the space-like subspace $\left(x^{1}, x^{2}, x^{3}, \tilde{x}^{0}\right)$, while being delocalized in the time-like subspace $\left(x^{0}, \tilde{x}^{1}, \tilde{x}^{2}, \tilde{x}^{3}\right)$. The field is confined within an infinitely long cylinder that extends along an $\left(x^{0}, \tilde{x}^{1}, \tilde{x}^{2}, \tilde{x}^{3}\right)$-direction. If the bradyon moves with a nonvanishing speed, the cylinder is suitably inclined. b) A tachyonic field can be localized within a region of the time-like subspace $\left(x^{0}, \tilde{x}^{1}, \tilde{x}^{2}, \tilde{x}^{3}\right)$, while being delocalized the spacelike subspace $\left(x^{1}, x^{2}, x^{3}, \tilde{x}^{0}\right)$. The field is now confined within a cylinder that is "orthogonal" to the cylinder of Fig. 3a. The case of a tachyon with infinite speed is illustrated. For finite tachyon speed the cylinder is suitably inclined.

where $\tilde{M}^{2}=-M^{2}>0$. This equation has the same form as Eq. (38), with $x^{\mu}$ and $\tilde{x}^{\mu}$ interchanged. Therefore, we can repeat the same procedure as before. A general solution is

$$
\tilde{\phi}=\int \mathrm{d}^{4} p \mathrm{~d}^{4} \tilde{p} \tilde{c}(p, \tilde{p}) \mathrm{e}^{i\left(-p_{\mu} x^{\mu}+\tilde{p}_{\mu} \tilde{x}^{\mu}\right)} \delta\left(-p^{\mu} p_{\mu}+\tilde{p}^{\mu} \tilde{p}_{\mu}-\tilde{M}^{2}\right)
$$

Now we choose a space-like component, e.g., $\tilde{p}^{0}$, and integrate it out. Instead of the solution (40), we obtain 5

$$
\begin{aligned}
\tilde{\phi}\left(x^{\mu}, \tilde{x}^{\mu}\right)=\int \mathrm{d} p^{0} \mathrm{~d}^{3} \mathbf{p} \mathrm{d}^{3} \tilde{\mathbf{p}} \frac{1}{2 \tilde{\omega}} & {\left[\mathrm{e}^{i\left(-p_{0} x^{0}+\mathbf{p x}+\tilde{\omega} \tilde{x}^{0}-\tilde{\mathbf{p}} \tilde{\mathbf{x}}\right)} \tilde{c}\left(p^{0}, \mathbf{p}, \tilde{\omega}, \tilde{\mathbf{p}}\right)\right.} \\
& \left.+\mathrm{e}^{i\left(-p_{0} x^{0}+\mathbf{p} \mathbf{x}-\tilde{\omega} \tilde{x}^{0}-\tilde{\mathbf{p}} \tilde{\mathbf{x}}\right)} \tilde{c}\left(p^{0}, \mathbf{p},-\tilde{\omega}, \tilde{\mathbf{p}}\right)\right],
\end{aligned}
$$

where

$$
\tilde{p}^{0}= \pm \tilde{\omega}, \quad \tilde{\omega}=\left|\sqrt{\left(p^{0}\right)^{2}-\mathbf{p}^{2}+\tilde{\mathbf{p}}^{2}+\tilde{M}^{2}}\right|
$$

If, instead of $\tilde{p}^{0}$, we integrate out from equation (54) some other space-like component, e.g., $p^{1} \equiv p_{x}$, then we obtain an expression, slightly different in form, but mathematically equivalent to the expression (55):

$$
\tilde{\phi}\left(x^{\mu}, \tilde{x}^{\mu}\right)=\int \mathrm{d} p^{0} \mathrm{~d} p^{2} \mathrm{~d} p^{3} \mathrm{~d} \tilde{p}^{0} \mathrm{~d} \tilde{\mathbf{p}} \frac{1}{2 \omega_{x}}\left[\mathrm{e}^{i\left(-p_{0}+\omega_{x} x^{1}+p_{2} x^{2}+p_{3} x^{3}-\tilde{p}_{0} \tilde{x}^{0}-\tilde{\mathbf{p}} \tilde{\mathbf{x}}\right)} \tilde{c}\left(p^{0}, \omega_{x}, p^{2}, p^{3}, \tilde{p}^{0}, \tilde{\mathbf{p}}\right)\right.
$$

${ }^{5}$ If we choose to integrate out the time-like component $p^{0}$, then we obtain

$\tilde{\phi}\left(x^{\mu}, \tilde{x}^{\mu}\right)=\int \mathrm{d}^{3} \mathbf{p} \mathrm{d} \tilde{p}^{0} \mathrm{~d}^{3} \tilde{\mathbf{p}} \frac{1}{2 \omega}\left[\mathrm{e}^{i\left(\omega x^{0}-\mathbf{p x}-\tilde{p}_{0} \tilde{x}^{0}+\tilde{\mathbf{p}} \tilde{\mathbf{x}}\right)} \tilde{c}\left(\omega, \mathbf{p}, \tilde{p}^{0}, \tilde{\mathbf{p}}\right)+\mathrm{e}^{i\left(-\omega \tilde{x}^{0}-\mathbf{p x}-\tilde{p}_{0} x^{0}+\tilde{\mathbf{p}} \tilde{\mathbf{x}}\right)} \tilde{c}\left(-\omega, \mathbf{p}, \tilde{p}^{0}, \tilde{\mathbf{p}}\right)\right]$,

where $p^{0}= \pm \omega$, and $\omega=\left|\sqrt{\mathbf{p}^{2}+\left(\tilde{p}^{0}\right)^{2}-\tilde{\mathbf{p}}^{2}-\tilde{M}^{2}}\right|$. 


$$
\left.+\mathrm{e}^{i\left(-p_{0}-\omega_{x} x^{1}+p_{2} x^{2}+p_{3} x^{3}-\tilde{p}_{0} \tilde{x}^{0}-\tilde{\mathbf{p}} \tilde{\mathbf{x}}\right)} \tilde{c}\left(p^{0},-\omega_{x}, p^{2}, p^{3}, \tilde{p}^{0}, \tilde{\mathbf{p}}\right)\right],
$$

where $p_{1}= \pm \omega_{x}, \quad \omega_{x}=\left|\sqrt{\left(p^{0}\right)^{2}-\left(p^{2}\right)^{2}-\left(p^{3}\right)^{2}-\left(\tilde{p}^{0}\right)^{2}+\left(\tilde{\mathbf{p}}^{2}\right)^{2}+\left(\tilde{\mathbf{p}}^{3}\right)^{2}+\tilde{M}^{2}}\right|$

A tachyonic field can be arbitrarily localized on the 4 -surface $\tilde{x}^{0}=0, \mathbf{x}=0$ that is spanned by time-like coordinates $\left(x^{0}, \tilde{x}^{1}, \tilde{x}^{2}, \tilde{x}^{3}\right)$, but it cannot be localized in the space-like directions $\left(x^{1}, x^{2}, x^{3}, \tilde{x}^{0}\right)$ (Fig. 3b).

For the particular choice of expansion coefficients,

$$
\begin{gathered}
c\left(p^{0}, \mathbf{p},-\tilde{\omega}, \tilde{\mathbf{p}}\right)=\delta\left(p^{2}\right) \delta\left(p^{3}\right) \delta\left(\tilde{p}^{0}\right) \delta\left(\tilde{p}^{1}\right) a\left(p^{0}, \omega_{x}, \tilde{p}^{2}, \tilde{p}^{3}\right), \\
c\left(p^{0}, \mathbf{p},-\tilde{\omega}, \tilde{\mathbf{p}}\right)=\delta\left(p^{2}\right) \delta\left(p^{3}\right) \delta\left(\tilde{p}^{0}\right) \delta\left(\tilde{p}^{1}\right) a\left(p^{0},-\omega_{x}, \tilde{p}^{2}, \tilde{p}^{3}\right),
\end{gathered}
$$

where $\omega_{x}=\left|\sqrt{\left(p^{0}\right)^{2}+\left(\tilde{\mathbf{p}}^{2}\right)^{2}+\left(\tilde{\mathbf{p}}^{3}\right)^{2}+\tilde{M}^{2}}\right|$, Eq. (157) becomes the tachyonic field (15)), considered in Sec. 2 .

Perhaps the fact that neither a bradyonic nor a tachyonic field can be freely localized on a 7D hypersurface, but there are restrictions on how they can be localized, should not be considered as problematic at all. In the literature it is often stated that the differential equations of physics are a powerful tool, but there is a problem, because we have no equations for initial data. The latter data are arbitrary, but it would be desirable to have a theory for initial data as well. We see that ultrahyperbolic equations, since they impose certain restriction on initial data, could be considered as equations that (at least partially) determine the initial data.

Under a SLT, the Klein-Gordon equation for a tachyonic field transforms into

$$
\left(-\partial^{\prime \mu} \partial_{\mu}^{\prime}+\tilde{\partial}^{\prime \mu} \tilde{\partial}_{\mu}^{\prime}-\tilde{M}^{2}\right) \tilde{\phi}^{\prime}\left(x^{\prime \mu}, \tilde{x}^{\prime \mu}\right)=0
$$

which is the Klein-Gordon equation for a bradyonic field $\phi\left(x^{\prime \mu}, \tilde{x}^{\prime \mu}\right)=\tilde{\phi}^{\prime}\left(x^{\prime \mu}, \tilde{x}^{\prime \mu}\right)$.

A field that is observed as tachyonic in a reference frame $S$, is observed as bradyonic in a superluminal reference frame $S^{\prime}$. In a given reference frame $S$ we have the bradyonic fields $\phi\left(x^{\mu}, \tilde{x}^{\mu}\right)$, satisfying $p^{\mu} p_{\mu}-\tilde{p}^{\mu} \tilde{p}_{\mu}>0$, and the tachyonic fields $\tilde{\phi}\left(x^{\mu} \tilde{x}^{\mu}\right)$, satisfying $p^{\mu} p_{\mu}-\tilde{p}^{\mu} \tilde{p}_{\mu}<0$. In a superluminal frame $S^{\prime}$, the type of those fields is interchanged.

We have a symmetry between bradyonic and tachyonic fields. None of those fields is more consistent than the other. Both kinds of fields are described by the ultrahyperbolic Klein-Gordon equation. We have reduced the problem of consistent propagating tachyonic fields to the problem of whether the ultrahyperbolic wave equation can make sense in physics. In addition, we have a problem of where do the extra dimensions $\tilde{x}^{\mu}, \mu=0,1,2,3$, come from and why do we not observe them. One possibility is just to suppose that our spacetime has not four, but eight dimensions (or, equivalently, that it is complex), and that the extra dimensions are not observed, because they are compactified. Another possibility is to consider the Clifford space, a $16 D$ manifold whose tangent space at any of its points is the Clifford algebra of spacetime $M_{1,3}$. 


\section{Polyvector-valued coordinates and momenta}

Geometric calculus based on Clifford algebra is a very successful mathematical tool for description of physics [41, 42]. In addition, it enables formulation of a new theory [18]-[28] in which the $4 D$ spacetime is replaced by a more general, $16 D$ space, called Clifford space, $C$. This is a space whose elements are not only points, but also oriented lines, 2-areas, 3 -volumes, and 4-volumes. Such generalization brings many new theoretical possibilities that are being explored [18]-[28].

The squared interval between two points in Minkowski space $M_{1,3}$ is given by the quadratic form

$$
Q=\left(x^{\mu}-x_{0}^{\mu}\right) \eta_{\mu \nu}\left(x^{\nu}-x_{0}^{\nu}\right) .
$$

If we take the square root, we obtain two possible results:

$$
\text { (i) } \sqrt{Q}=\Delta s,
$$

where $\Delta s$ is the scalar distance, and

$$
\text { (ii) } \sqrt{Q}=\Delta x=\left(x^{\mu}-x_{0}^{\mu}\right) \gamma_{\mu},
$$

where $\Delta x$ is the vector that joins the points $x^{\mu}$ and $x_{0}^{\mu}$. Here the basis vectors $\gamma_{\mu}$ are generators of the Clifford algebra, $C l(1,3)$, satisfying

$$
\gamma_{\mu} \cdot \gamma_{\nu} \equiv \frac{1}{2}\left(\gamma_{\mu} \gamma_{\nu}+\gamma_{\nu} \gamma_{\mu}\right)=\eta_{\mu \nu}
$$

The points of $M_{1,3}$ can be described by vectors $x=x^{\mu} \gamma_{\mu}$, originating from a chosen fixed

point, $x_{0}^{\mu}=0$. The $4 D$ space of vectors $x^{\mu} \gamma_{\mu}$ is a subspace of the $16 D$ Clifford algebra $C l(1,3)$, whose elements are polyvectors

$$
X=\sigma \mathbf{1}+x^{\mu} \gamma_{\mu}+x^{\mu \nu} \gamma_{\mu} \wedge \gamma_{\nu}+\tilde{x}^{\mu} \gamma_{5} \gamma_{\mu}+\tilde{\sigma} \gamma_{5} \equiv x^{M} \gamma_{M}
$$

Here, $\gamma_{5}=\gamma_{0} \gamma_{1} \gamma_{2} \gamma_{3}$, and ' $\wedge$ ' the wedge product, e.g., $\gamma_{\mu} \wedge \gamma_{\nu}=(1 / 2)\left(\gamma_{\mu} \gamma_{\nu}-\gamma_{\nu} \gamma_{\mu}\right)$. Polyvectors, or $r$-vectors, describe oriented $r$-volumes (called also $r$-areas), $r=0,1,2,3,4$. The idea is 23]-25] that $r$-volumes are associated with extended events living in spacetime $M_{1,3}$. Thus, e.g., 2 -volumes that are described by $x^{\mu \nu} \gamma_{\mu} \wedge \gamma_{\nu}$ are associated with the class of those physical objects, whose representative is a closed instantonic string, or alternatively, and open instantonic 2-brane, depending on whether the scalar $\sigma$ is zero, or different from zero. Namely, the scalar $\sigma$ can be defined in terms of the length, area, 3-volume, and 4volume of, respectively, the open 1-brane, 2-brane, 3-brane and 4-brane. For instance, for an open instantonic string (i.e., 1-brane), the scalar is defined as

$$
\sigma_{1}=\frac{\mathcal{A}_{1}}{L_{P}}=\frac{1}{L_{P}} \int \mathrm{d} \xi\left(\frac{\partial X^{\mu}}{\partial \xi} \frac{\partial X^{\nu}}{\partial \xi} \eta_{\mu \nu}\right)^{1 / 2}
$$


where $\mathcal{A}_{1}$ is the string length, and $\xi$ an arbitrary real parameter denoting the points on the string. In general, for an open instantonic $r_{\sigma}$-brane, we have

$$
\sigma_{r_{\sigma}}=\frac{\mathcal{A}_{r_{\sigma}}}{\left(L_{P}\right)^{r_{\sigma}}}=\frac{1}{\left(L_{P}\right)^{r_{\sigma}}} \int \mathrm{d} \xi^{1} \mathrm{~d} \xi^{2} \ldots \mathrm{d} \xi^{r_{\sigma}} \sqrt{\operatorname{det} \frac{\partial X^{\mu}}{\partial \xi^{a}} \frac{\partial X^{\nu}}{\partial \xi^{b}} \eta_{\mu \nu}}
$$

where $\mathcal{A}_{r_{\sigma}}$ is the $r_{\sigma}$-dimensional area/volume of the brane, and $\xi^{a}$ or $\xi^{b}, a, b=1,2, \ldots, r_{\sigma}$, the parameters (coordinates) denoting the points of the brane. Here, $L_{P}$ is a fundamental length, for instance the Planck length,

For a generic object, that is a conglomerate of instantonic $r_{\sigma}$-branes, for various values of $r_{\sigma}=1,2,3,4$, the scalar is defined as the sum

$$
\sigma=\sum_{r_{\sigma}=1}^{4} \frac{\mathcal{A}_{r_{\sigma}}}{\left(L_{P}\right)^{r_{\sigma}}}
$$

The scalar $\sigma$ thus determines to what extent the object, described by a polyvector (65), and envisaged as a conglomerate of instantonic $r$-branes, $r=0,1,2,3,4$, contains closed $\left(r_{\sigma}-1\right)$-branes and open $r_{\sigma}$-branes, $r_{\sigma}=1,2,3,4$. If $\sigma=0$, then only closed $\left(r_{\sigma}-1\right)$-branes are present.

Our objects are instantonic $r$-branes, which mean that they are localized in spacetime 6 They are a generalization of the concept of an 'event' to which there corresponds a point in spacetime. Instead of a point, we have now in general a set of $r$-volumes, $x^{\mu_{1} \ldots \mu_{r}}$, $r=0,1,2,3,4$, that describe an extended event in spacetime 7 The space of the extended events is called Clifford space, $C$. It is manifold whose tangent space at any of its points is a Clifford algebra $C l(1,3)$. If $C$ is a flat space, then it is isomorphic to the Clifford algebra with elements $X=x^{\mu_{1} \ldots \mu_{r}} \gamma_{\mu_{1} \ldots \mu_{r}} \equiv x^{M} \gamma_{M}$ (Eq. (65)).

Instead of the relativity in spacetime, we have now the relativity in Clifford space [18][28]. The line element is

$$
\mathrm{d} S^{2}=\mathrm{d} x^{M} \mathrm{~d} x^{N} G_{M N},
$$

where the metric is defined in terms of the scalar product between two basis elements:

$$
G_{M N}=\gamma_{M}^{\ddagger} * \gamma_{N}=\left\langle\gamma_{M}^{\ddagger} \gamma_{N}\right\rangle_{0}
$$

Here \langle\rangle$_{0}$ denotes the scalar part, and ' $\ddagger$ ' reversion, so that $\gamma_{M}^{\ddagger} \equiv\left(\gamma_{\mu_{1}} \gamma_{\mu_{2}} \ldots \gamma_{\mu_{r}}\right)^{\ddagger}=$ $\gamma_{\mu_{r}} \gamma_{\mu_{r}-1} \ldots \gamma_{\mu_{2}} \gamma_{\mu_{1}}$. From the definition (70) we obtain the following explicit form for the line element:

$$
\mathrm{d} S^{2}=\mathrm{d} \sigma^{2}+\left(\mathrm{d} x^{0}\right)^{2}-\left(\mathrm{d} x^{1}\right)^{2}-\left(\mathrm{d} x^{2}\right)^{2}-\left(\mathrm{d} x^{3}\right)^{2}-\left(\mathrm{d} x^{01}\right)^{2}-\left(\mathrm{d} x^{02}\right)^{2}-\left(\mathrm{d} x^{03}\right)^{2}
$$

\footnotetext{
${ }^{6}$ The usual $p$-branes are localized in space, but they are infinitely extended into a time-like direction; therefore, they are $(p+1)$-dimensional worldsheets in spacetime.

${ }^{7}$ Polyvector coordinates $x^{\mu_{1} \ldots \mu_{r}}$ describe a class of $r$-branes, all having the same $x^{\mu_{1} \ldots \mu_{r}}$. See Ref. [25, 27].
} 


$$
+\left(\mathrm{d} x^{12}\right)^{2}+\left(\mathrm{d} x^{13}\right)^{2}+\left(\mathrm{d} x^{23}\right)^{2}-\left(\mathrm{d} \tilde{x}^{0}\right)^{2}+\left(\mathrm{d} \tilde{x}^{1}\right)^{2}+\left(\mathrm{d} \tilde{x}^{2}\right)^{2}+\left(\mathrm{d} \tilde{x}^{3}\right)^{2}-\mathrm{d} \tilde{\sigma}^{2} .
$$

We see that the signature is $(8,8)$.

Coordinates $x^{M}$ denote a point in Clifford space, $C$. A point in $C$ is associated with an event in $C$, which in turn corresponds to an extended event in spacetime $M_{1,3}$. Let us now consider a family of events, described by a worldline $x^{M}=X^{M}(\zeta)$ in $C$, where $\zeta$ is an arbitrary monotonically increasing parameter along the worldline. We assume that such a wordline, if associated with a physical object living in $C$, satisfies the "minimal" length action

$$
I\left[X^{M}\right]=M \int \mathrm{d} \zeta\left(\dot{X}^{M} \dot{X}^{N} G_{M N}\right)^{1 / 2},
$$

where $\dot{X}^{M} \equiv \mathrm{d} X^{M} / \mathrm{d} \zeta$. This is an action for a point particle in $C$. In spacetime $M_{1,3}$, such particle is extended and traces a "thick" worldline.

The action (72) is invariant under reparametrizations of $\zeta$. As a consequence, the canonical momenta $P_{M}=\partial L / \partial \dot{X}^{M}=M \dot{X}^{M} /\left(\dot{X}^{N} \dot{X}_{N}\right)^{1 / 2}$ satisfy the constraint

$$
G^{M N} P_{M} P_{N}-M^{2}=0
$$

\subsection{Generalized Klein-Gordon equation}

Upon quantization, the constraint (73) becomes the ultrahyperbolic Klein-Gordon equation

$$
\left(G^{M N} \partial_{M} \partial_{N}+M^{2}\right) \phi\left(x^{M}\right)=0
$$

or explicitly,

$$
\left(\frac{\partial^{2}}{\partial \sigma^{2}}+\partial^{\mu} \partial_{\mu}-\tilde{\partial}^{\mu} \tilde{\partial}_{\mu}+\partial^{\mu \nu} \partial_{\mu \nu}-\frac{\partial^{2}}{\partial \tilde{\sigma}^{2}}+M^{2}\right) \phi\left(\sigma, x^{\mu}, x^{\mu \nu}, \tilde{x}^{\mu}, \tilde{\sigma}\right)=0,
$$

which is of the same form as Eq. (38), except that now the space is 16-dimensional, with signature $(8,8)$. The coordinates are $x^{M}=\left(\sigma, x^{\mu}, x^{\mu \nu}, \tilde{x}^{\mu}, \tilde{\sigma}\right)$, and the metric is $G_{M N}$, given by Eq. (71).

If we introduce the new coordinates

$$
\tau=\frac{1}{\sqrt{2}}(\tilde{\sigma}-\sigma), \quad \lambda=\frac{1}{\sqrt{2}}(\tilde{\sigma}+\sigma),
$$

the Klein-Gordon equation becomes

$$
\left(-2 \frac{\partial^{2}}{\partial \tau \partial \lambda}+\partial^{\mu} \partial_{\mu}-\tilde{\partial}^{\mu} \tilde{\partial}_{\mu}+\partial^{\mu \nu} \partial_{\mu \nu}+M^{2}\right) \psi\left(\tau, \lambda, x^{\mu}, \tilde{x}^{\mu}, x^{\mu \nu}\right)=0 .
$$

We will write this compactly as

$$
\left(-2 \partial_{\tau} \partial_{\lambda}+G^{\bar{\mu} \bar{\nu}} \partial_{\bar{\mu}} \partial_{\bar{\nu}}+M^{2}\right) \psi\left(\tau, \lambda, x^{\bar{\mu}}\right)=0,
$$

where $x^{\bar{\mu}}=\left(x^{\mu}, \tilde{x}^{\mu}, x^{\mu \nu}\right)$, and $G^{\bar{\mu} \bar{\nu}}$ the metric with signature $(7,7)$. 
A general solution of Eq. (78) is

$$
\psi=\int \mathrm{d} p_{\tau} \mathrm{d} p_{\lambda} \mathrm{d}^{14} \bar{p} c\left(p_{\tau}, p_{\lambda}, p_{\bar{\mu}}\right) \mathrm{e}^{i\left(p_{\tau} \tau+p_{\lambda} \lambda+p_{\bar{\mu}} x^{\bar{\mu}}\right)} \delta\left(2 p_{\tau} p_{\lambda}-G^{\bar{\mu} \bar{\nu}} p_{\bar{\mu}} p_{\bar{\nu}}+M^{2}\right) .
$$

Performing the integration over $p_{\tau}$, we have

$$
\psi=\int \mathrm{d} p_{\lambda} \mathrm{d}^{14} \bar{p} a\left(\omega, p_{\lambda}, p_{\bar{\mu}}\right) \mathrm{e}^{i \omega \tau} \mathrm{e}^{i p_{\bar{\mu}} x^{\bar{\mu}}} \mathrm{e}^{i p_{\lambda} \lambda},
$$

where

$$
\omega=\frac{1}{2 p_{\lambda}}\left(G^{\bar{\mu} \bar{\nu}} p_{\bar{\mu}} p_{\bar{\nu}}-M^{2}\right) .
$$

and

$$
a\left(\omega, p_{\lambda}, p_{\bar{\mu}}\right) \equiv \frac{1}{2 p_{\lambda}} c\left(\omega, p_{\lambda}, p_{\bar{\mu}}\right) .
$$

All values of $p_{\lambda}, p_{\bar{\mu}}$ between $-\infty$ and $+\infty$ are allowed. The initial data, given at $\tau=0$, are

$$
\begin{aligned}
& \psi\left(\tau=0, \lambda, x^{\bar{\mu}}\right)=\int \mathrm{d} p_{\lambda} \mathrm{d}^{14} \bar{p} a\left(\omega, p_{\lambda}, p_{\bar{\mu}}\right) \mathrm{e}^{i p_{\bar{\mu}} x^{\bar{\mu}}} \mathrm{e}^{i p_{\lambda} \lambda}, \\
& \dot{\psi}\left(\tau=0, \lambda, x^{\bar{\mu}}\right)=\int \mathrm{d} p_{\lambda} \mathrm{d}^{14} \bar{p} i \omega a\left(\omega, p_{\lambda}, p_{\bar{\mu}}\right) \mathrm{e}^{i p_{\bar{\mu}} x^{\bar{\mu}}} \mathrm{e}^{i p_{\lambda} \lambda} .
\end{aligned}
$$

The latter equations tell us that $\psi\left(\tau=0, \lambda, x^{\bar{\mu}}\right)$ and $\dot{\psi}\left(\tau=0, \lambda, x^{\bar{\mu}}\right)$ are expanded in terms of the complete set of functions $\exp \left[i\left(p_{\lambda} \lambda+p_{\bar{\mu}} x^{\bar{\mu}}\right)\right]$, and that there is no restriction on initial data, except that $\psi$ must be normalizable according to $\int \mathrm{d} \lambda \mathrm{d}^{14} \bar{x}|\psi|^{2}=1$. By the Fourier transform, the expansion coefficients $a\left(\omega, p_{\lambda}, p_{\bar{\mu}}\right)$ can be expressed in terms of the initial data. As a consequence, $\psi\left(\tau, \lambda, x^{\bar{\mu}}\right)$ is uniquely determined at any $\tau>0$. The Cauchy problem is thus well defined 8 .

If we take

$$
a\left(\omega, p_{\lambda}, p_{\bar{\mu}}\right)=\delta\left(p_{\lambda}-\Lambda\right) A\left(p_{\bar{\mu}}\right)
$$

then

$$
\psi\left(\tau, \lambda, x^{\bar{\mu}}\right)=\int \mathrm{d}^{14} \bar{p} A\left(p_{\bar{\mu}}\right) \mathrm{e}^{i \omega \tau} \mathrm{e}^{i p_{\bar{\mu}} x^{\bar{\mu}}} \mathrm{e}^{i \Lambda \lambda} \equiv \varphi\left(\tau, x^{\bar{\mu}}\right) \mathrm{e}^{i \Lambda \lambda}
$$

where

$$
\omega=\frac{1}{2 \Lambda}\left(G^{\bar{\mu} \bar{\nu}} p_{\bar{\mu}} p_{\bar{\nu}}-M^{2}\right) .
$$

This is a solution of Eq. (178) with the definite value $p_{\lambda}=\Lambda$, of the momentum operator $\hat{p}_{\lambda}=-i \partial_{\lambda}$. By plugging Eq. (866) into Eq. (78), we obtain the wave equation for $\varphi\left(\tau, x^{\bar{\mu}}\right)$ :

$$
i \frac{\partial \varphi\left(\tau, x^{\bar{\mu}}\right)}{\partial \tau}=\frac{1}{2 \Lambda}\left(G^{\bar{\mu} \bar{\nu}} \partial_{\bar{\mu}} \partial_{\bar{\nu}}+M^{2}\right) \varphi\left(\tau, x^{\bar{\mu}}\right) .
$$

${ }^{8}$ That the Cauchy problem for an ultrahyperbolic partial differential equation is well defined for initial data, taken at a light-like hypersurface, is a known result [17. 
This is the generalized 9 Stueckleberg equation for the wave function $\varphi\left(\tau, x^{\bar{\mu}}\right)$. It is like the Schrödinger equation, the evolution parameter being $\tau$, and the wave function being normalized according to $\int \mathrm{d}^{14} \bar{x}\left|\varphi\left(\tau, x^{\bar{\mu}}\right)\right|^{2}=1$.

From the wave equation (88) and its solution (86) we see that $M^{2}$ gives a constant phase factor $\exp \left[-i(1 / 2 \Lambda) M^{2} \tau\right]$, and has no other role. It makes not much difference if $M^{2}>0$ or $M^{2}<0$. The solution of Eq. (88) is thus not sensitive on whether the field is bradyonic or tachonic in the $16 D$ space $C$.

On the other hand, it is well-known that the Stueckelberg [43, 44] theory admits superluminal motion in $4 D$ spacetime $M_{1,3}$. All 14 momenta $p_{\bar{\mu}}=\left(p_{\mu}, \tilde{p}_{\bar{\mu}}, p_{\mu \nu}\right)$ occurring in the solution (86) are independent, and their quadratic form $G^{\bar{\mu} \bar{\nu}} p_{\bar{\mu}} p_{\bar{\nu}} \equiv \bar{p}^{2}$ can be either positive or negative. The same is true for the quadratic form of the spacetime momenta, $\eta^{\mu \nu} p_{\mu} p_{\nu} \equiv p^{2}$. It can be $p^{2}>0$, in which case the field behaves as subluminal in $M_{1,3}$. But it also can be $p^{2}<0$, and then the field is tachyonic in $M_{1,3}$. In the presence of suitable interaction 10 , there can be smooth transition from the subluminal to the superluminal case.

\subsection{Generalized Dirac equation}

In Refs. 21, 22] the following generalization of the Dirac equation for a polyvector valued wave funtion was proposed:

$$
\gamma^{M} \partial_{M} \Phi=0
$$

where $\Phi=\phi^{A} \gamma_{A}=\phi^{\tilde{A}} \xi_{\tilde{A}}$ is expanded in terms of the Clifford algebra basis, $\gamma_{A}=\left(1, \gamma_{a}, \gamma_{a} \wedge\right.$ $\left.\gamma_{b}, \ldots\right)$, or in terms pf the generalized spinor basis $\xi_{\tilde{A}} \equiv \xi_{\alpha i}$. Here $\alpha=1,2,3,4$ is the spinor index, and $i=1,2,3,4$ the index denoting four minimal left ideals of $C l(1,3)$, a tangent space of the Clifford space, $C$. The components $\phi^{\tilde{A}}=\phi^{\tilde{A}}\left(x^{M}\right)$ are complex valued fields on $C$.

Explicitly, Eq. (89) reads

$$
\left(\frac{\partial}{\partial \sigma}+\gamma^{\mu} \partial_{\mu}+\gamma^{\mu} \gamma^{\nu} \partial_{\mu \nu}+\gamma^{5} \gamma^{\mu} \tilde{\partial}_{\mu}+\gamma^{5} \frac{\partial}{\partial \tilde{\sigma}}\right) \Phi=0
$$

Let us now consider two possible kinds of solutions, each satisfying a different Ansatz. Ansatz I

$$
\begin{aligned}
& \Phi=\mathrm{e}^{i m \sigma} \mathrm{e}^{i \tilde{m} \tilde{\sigma}} \Psi\left(x^{\mu}, x^{\mu \nu}, \tilde{x}^{\mu}\right) \\
& \frac{\partial \Phi}{\partial \sigma}=i m \Phi, \quad \frac{\partial \Phi}{\partial \tilde{\sigma}}=i \tilde{m} \Phi
\end{aligned}
$$

\footnotetext{
${ }^{9}$ The usual Stueckelberg equation [43, 44] involves 4 spacetime coordinates $x^{\mu}, \mu=0,1,2,3$, whereas the generalized equation (88) involves 14 coordinates $x^{\bar{\mu}}=\left(x^{\mu}, \tilde{x}^{\mu}, x^{\mu \nu}\right)$.

${ }^{10} \mathrm{An}$ example of an interaction that can accelerate a classical Stueckelberg point particle from subluminal to superluminal speeds is considered in Ref. [28]. See also Refs. [43, 44].
} 
Then Eq. (90) becomes

$$
\left(\gamma^{\mu} \partial_{\mu}+\gamma^{\mu} \gamma^{\nu} \partial_{\mu \nu}+\gamma^{5} \gamma^{\mu} \tilde{\partial}_{\mu}+i m+i \tilde{m} \gamma^{5}\right) \Psi=0
$$

Ansatz II

$$
\begin{aligned}
& \Phi=\mathrm{e}^{-m \sigma} \mathrm{e}^{-\tilde{m} \tilde{\sigma}} \Psi\left(x^{\mu}, x^{\mu \nu}, \tilde{x}^{\mu}\right) \\
& \frac{\partial \Phi}{\partial \sigma}=-m \Phi, \quad \frac{\partial \Phi}{\partial \tilde{\sigma}}=-\tilde{m} \Phi
\end{aligned}
$$

Then Eq. (90) becomes

$$
\left(\gamma^{\mu} \partial_{\mu}+\gamma^{\mu} \gamma^{\nu} \partial_{\mu \nu}+\gamma^{5} \gamma^{\mu} \tilde{\partial}_{\mu}-m-\tilde{m} \gamma^{5}\right) \Psi=0
$$

A special case of the latter equation is

$$
\left(\gamma^{\mu} \partial_{\mu}-\tilde{m} \gamma^{5}\right) \Psi
$$

which holds when $\Psi$ does not depend on $x^{\mu \nu}, \tilde{x}^{\mu}$, and $\sigma$. Multiplying (97) from the left by $\gamma^{5}$, we obtain

$$
\left(\gamma^{5} \gamma^{\mu} \partial_{\mu}+\tilde{m}\right) \Psi=0
$$

Further we have

$$
\left(\left(\gamma^{5} \gamma^{\mu}\right)^{\ddagger} \partial_{\mu}+\tilde{m}\right)\left(\gamma^{5} \gamma^{\mu} \partial_{\mu}+\tilde{m}\right) \Psi=\left(-\eta^{\mu \nu} \partial_{\mu} \partial_{\nu}+\tilde{m}^{2}\right) \Psi=0 .
$$

This is the "tachyonic" Klein-Gordon equation11, satisfying the 4-momentum constraint $p^{\mu} p_{\mu}=-\tilde{m}^{2}$. The same constraint holds for Eq. (98), which is therefore the "tachyonic" Dirac equation. Such equation 12 was proposed by Chodos et al. [29]. It is a special case of the generalized Dirac equation (89). Solutions of Eq. (98) and their localizability were investigated in Ref. [45]. A different approach to a tachyonic Dirac equation is considered in Ref. [46]. The imaginary mass Dirac equation and its quantization has been investigated by Jentschura [4] who found that right handed states acquire a negative Fock-space norm, which could explain the absence of right-handed neutrinos.

If we start from the original equation (89), multiply it by $\gamma^{M^{\ddagger}}$ and take the scalar part, we have

$$
\left\langle\gamma^{M \ddagger} \gamma^{N}\right\rangle_{0} \partial_{M} \partial_{N} \Phi=0, \quad \text { i.e., } \quad G^{M N} \partial_{M} \partial_{N} \Phi=0,
$$

or explicitly,

$$
\left(\frac{\partial^{2}}{\partial \sigma^{2}}+\eta^{\mu \nu} \partial_{\mu} \partial_{\nu}+\partial^{\mu \nu} \partial_{\mu \nu}-\eta^{\mu \nu} \tilde{\partial}_{\mu} \tilde{\partial}_{\nu}-\frac{\partial^{2}}{\partial \tilde{\sigma}^{2}}\right) \Phi=0,
$$

\footnotetext{
${ }^{11}$ As discussed in Sec. 2, this is not the true tachyonic Klein-Gordon equation, because it cannot be obtained from a bradyonic equation by a SLT. It is a part of the complete equation (103), which is either tachyonic or bradyonic, depending on the sign of $m^{2}-\tilde{m}^{2}$.

${ }^{12}$ We use here the definition $\gamma^{5}=\gamma^{0} \gamma^{1} \gamma^{2} \gamma^{3}$, satisfying $\left(\gamma^{5}\right)^{2}=-1,\left(\gamma^{5}\right)^{\ddagger}=\gamma^{5}$, that is customary in the literature on Clifford algebras. Chodos et al. 29] use the definition $\gamma^{5}=i \gamma^{0} \gamma^{1} \gamma^{2} \gamma^{3}$, satisfying $\left(\gamma^{5}\right)^{2}=1,\left(\gamma^{5}\right)^{\ddagger}=-\gamma^{5}, i^{\ddagger}=-1$.
} 
which is Eq. (75) for $M=0$.

Eq. (101), in the case of Ansatz I becomes

$$
\left(\partial^{\mu} \partial_{\nu}+\partial^{\mu \nu} \partial_{\mu \nu}-\tilde{\partial}^{\mu} \tilde{\partial}_{\nu}-m^{2}+\tilde{m}^{2}\right) \Psi=0,
$$

and in the case of Ansatz II, it becomes

$$
\left(\partial^{\mu} \partial_{\nu}+\partial^{\mu \nu} \partial_{\mu \nu}-\tilde{\partial}^{\mu} \tilde{\partial}_{\nu}+m^{2}-\tilde{m}^{2}\right) \Psi=0,
$$

Both equations, (102), (103), are either bradyonic or tachyonic, depending on the sign of $m^{2}-\tilde{m}^{2}$.

Besides Ansatz I and Ansatz II, we can also consider

Ansatz III

$$
\Phi=\mathrm{e}^{-m \sigma} \mathrm{e}^{i \tilde{m} \tilde{\sigma}} \Psi\left(x^{\mu}, x^{\mu \nu}, \tilde{x}^{\mu}\right)
$$

with

$$
\begin{gathered}
\left(\gamma^{\mu} \partial_{\mu}+\gamma^{\mu} \gamma^{\nu} \partial_{\mu \nu}+\gamma^{5} \gamma^{\mu} \tilde{\partial}_{\mu}-m+i \tilde{m} \gamma^{5}\right) \Psi=0 \\
\left(\partial^{\mu} \partial_{\nu}+\partial^{\mu \nu} \partial_{\mu \nu}-\tilde{\partial}^{\mu} \tilde{\partial}_{\mu}+m^{2}+\tilde{m}^{2}\right) \Psi=0
\end{gathered}
$$

and

Ansatz IV

$$
\Phi=\mathrm{e}^{i m \sigma} \mathrm{e}^{-\tilde{m} \tilde{\sigma}} \Psi\left(x^{\mu}, x^{\mu \nu}, \tilde{x}^{\mu}\right)
$$

with

$$
\begin{gathered}
\left(\gamma^{\mu} \partial_{\mu}+\gamma^{\mu} \gamma^{\nu} \partial_{\mu \nu}+\gamma^{5} \gamma^{\mu} \tilde{\partial}_{\mu}+i m-\tilde{m} \gamma^{5}\right) \Psi=0 . \\
\left(\partial^{\mu} \partial_{\nu}+\partial^{\mu \nu} \partial_{\mu \nu}-\tilde{\partial}^{\mu} \tilde{\partial}_{\mu}-m^{2}-\tilde{m}^{2}\right) \Psi=0
\end{gathered}
$$

For real $m, \tilde{m}$, Eqs. (105),(106) are bradyonic, whereas Eqs. (108), (109) are tachyonic.

If $\partial_{\mu \nu} \Psi=0, \tilde{\partial}_{\mu} \Psi=0$, then Eqs. (105), (108) become

$$
\begin{aligned}
& \left(\gamma^{\mu} \partial_{\mu}-m+i \tilde{m} \gamma^{5}\right) \Psi=0 \\
& \left(\gamma^{\mu} \partial_{\mu}+i m-\tilde{m} \gamma^{5}\right) \Psi=0
\end{aligned}
$$

Equations of the latter kind were considered in Refs. [30].

Usage of the solutions with $\operatorname{Exp}[-m \sigma]$ and $\operatorname{Exp}[-\tilde{m} \tilde{\sigma}]$ makes sense only, if the boundary conditions in the space $(\sigma, \tilde{\sigma})$ are such that they prevent the escape of functions into infinity.

Our choice of basis,

$$
\gamma_{M}=\left(1, \gamma_{\mu}, \gamma_{\mu} \wedge \gamma_{\mu}, \gamma^{5} \gamma_{\mu}, \gamma^{5}\right)
$$

leads to Eqs. (93), (96), (105), (108), corresponding to the choices Eqs. (91), (94) (104), (107), respectively. We have seen that Eqs. (96) and (108) contain the so called "tachyonic" equation (97) of Chodos et al. [29]. 
Eqs. (96) and (105) contain, as a special case, the following equation:

$$
\left(\gamma^{\mu} \partial_{\mu}-m\right) \Psi=0
$$

which, when squared according to (100), gives the bradyonic Klein-Gordon equation. But Eq. (113) is not quite the usual Dirac equation, because an $i$ is missing. It is straightforward to verify that an alternative choice of basis, namely

$$
\gamma_{M}=\left(-i, \gamma_{\mu}, \gamma_{\mu} \wedge \gamma_{\mu}, \gamma^{5} \gamma_{\mu}, \gamma^{5}\right)
$$

gives the ordinary Dirac equation,

$$
\left(i \gamma^{\mu} \partial_{\mu}-m\right) \Psi=0
$$

which when squared according to (100), and taking into account $i^{\ddagger}=-i$, also gives the bradyonic Klein-Gordon equation.

With the basis (114), instead of Eqs. (110), (111), we have

$$
\begin{gathered}
\left(\gamma^{\mu} \partial_{\mu}+i m+i \tilde{m} \gamma^{5}\right) \Psi=0 \\
\left(\gamma^{\mu} \partial_{\mu}+m-\tilde{m} \gamma^{5}\right) \Psi=0
\end{gathered}
$$

which, when multiplied by $i$, are the generalized bradyonic and tachyonic Dirac equations studied in Ref. [30].

To avoid problems with localizability of the field $\Psi$, let us again consider the light cone like coordinates (76). In such coordinates the generalized Dirac equation (90) reads

$$
-\frac{1}{\sqrt{2}}\left(1-\gamma^{5}\right) \partial_{\tau} \Psi+\frac{1}{\sqrt{2}}\left(1+\gamma^{5}\right) \partial_{\lambda} \Psi+\gamma^{\bar{\mu}} \partial_{\bar{\mu}} \Psi=0 .
$$

Using

$$
\begin{aligned}
& \frac{1}{\sqrt{2}}\left(1+\gamma^{5}\right) \frac{1}{\sqrt{2}}\left(1-\gamma^{5}\right)=1, \\
& \frac{1}{\sqrt{2}}\left(1+\gamma^{5}\right) \frac{1}{\sqrt{2}}\left(1+\gamma^{5}\right)=\gamma^{5},
\end{aligned}
$$

Eq. (118) can be rewritten in the form

$$
-\partial_{\tau} \Psi+\gamma^{5} \partial_{\lambda} \Psi+\frac{1}{\sqrt{2}}\left(1+\gamma^{5}\right) \gamma^{\bar{\mu}} \partial_{\bar{\mu}} \Psi=0 .
$$

Eqs. (118), (121) satisfy the equation

$$
\left(-2 \partial_{\tau} \partial_{\lambda}+\partial^{\bar{\mu}} \partial_{\bar{\mu}}\right) \Psi=0,
$$

which is Eq. (78) with $M=0$. 
If $\Psi$ is an eigenstate of the operator $-i \partial_{\lambda}$, so that $-i \partial_{\lambda} \Psi=\Lambda \Psi$, then Eq. (121) becomes

$$
\left(-\partial_{\tau}+i \Lambda \gamma^{5}+\frac{1}{\sqrt{2}}\left(1+\gamma^{5}\right) \gamma^{\bar{\mu}} \partial_{\bar{\mu}}\right) \Psi=0
$$

Here $\tau$ is the Stueckelberg evolution parameter. In principle, solutions of Eq. (123) should be found in analogous way as solutions of Eq. (78), by taking into account the dispersion relation $-2 p_{\tau} p_{\lambda}+p^{\bar{\mu}} p_{\bar{\mu}}=0$ that comes from Eq. (122). However, a difference is that now $\Psi$ is a generalized spinor field. It is localized on a 15-dimensional hypersurface $\tau=$ constant, spanned by coordinates $\lambda, x^{\bar{\mu}}$, where $x^{\bar{\mu}}=\left(x^{\mu}, \tilde{x}^{\mu}, x^{\mu \nu}\right)$.

If $\Psi$ is also an eigenstate of $-i \partial_{\tau}$, so that $-i \partial_{\tau} \Psi=K \Psi$, then we obtain the equation

$$
\left(-i K+i \Lambda \gamma^{5}+\frac{1}{\sqrt{2}}\left(1+\gamma^{5}\right) \gamma^{\bar{\mu}} \partial_{\bar{\mu}}\right) \Psi=0,
$$

or, equivalently (c.f., Eq. (118)),

$$
\left(-\frac{1}{\sqrt{2}}\left(1-\gamma^{5}\right) i K+\frac{1}{\sqrt{2}}\left(1+\gamma^{5}\right) i \Lambda+\gamma^{\bar{\mu}} \partial_{\bar{\mu}}\right) \Psi=0 .
$$

If in the latter equation we rearrange the terms and set $m=(1 / \sqrt{2})(\Lambda-K), \quad \tilde{m}=$ $(1 / \sqrt{2})(\Lambda+K)$, then we obtain Eq. (93), which confirms the consistency of the procedure. If the eigenvalues $K$ and $\Lambda$ are real or imaginary, then we reproduce Eqs. (96), (105) and (108). Special cases of the last two equations are (110), (111), that in terms of the alternative Clifford algebra basis (114), can be written in the form (116),(117). Solutions of the latter equations and their physical implications have been considered by Jentschura and Wundt 30].

\section{Discussion}

We have reduced the problem of consistent propagating tachyonic field to the problem of whether the Stueckelberg field makes sense in physics. We assumed that physics has to be formulated in the $16 D$ Clifford space $C$, a manifold whose tangent space at any point is Clifford algebra. Since the signature of $C$ is $(8,8)$, we arrived upon quantization at the ultrahyperbolic Klein-Gordon or Dirac equation. The Cauchy problem for such equation is not well posed, unless we take initial data on a light-like hypersurface. Then, after using the light cone coordinates, such that the evolution parameter $\tau$ is a superposition of the scalar and pseudoscalar coordinate, the Klein-Gordon and the Dirac equation become the corresponding generalized Stueckelberg equations. A different choice of light cone coordinates, involving the time-like coordinate $x^{0}$, although mathematically admissible, would be physically problematic, because we do not live on a light cone of $M_{1,3}$. A virtue of the Clifford space is that it contains extra time-like and space-like dimensions, besides the ordinary $x^{\mu}, \mu=0,1,2,3$. Therefore, in $C$ there exist light-like hypersurfaces that do not 
involve $x^{\mu}$, and one can pose the initial value problem on one of such hypersurfaces. However, the evolution parameter is then not $x^{0}$, but some other parameter associated with a family of hypersurfaces. In our case, such a parameter is $\tau$, the light cone coordinate (76). According to this theory, we live on a light-like hypersurface in Clifford space, and experience an evolution whose parameter is $\tau$. The coordinate $x^{0}$ has now the same status as the spatial coordinates $x^{i}, i=1,2,3$. The $x^{i}$ of a physical object change with evolution, and so does $x^{0}$. The wave packet can now be localized not only around $x^{i}$, but also around $x^{0}$, i.e., around a point ("event") $\mathcal{P}$ in $M_{1,3}$. And with increasing $\tau$, the point $\mathcal{P}(\tau)$ moves in $M_{1,3}$. Since the latter space is a subspace of the $16 D$ Clifford space $C$, we have in fact a wave packet localized around a point $\mathcal{E}$, determined by the coordinates $x^{\bar{\mu}}=\left(x^{\mu}, \tilde{x}^{\mu}, x^{\mu \nu}\right)$ of the $14 D$ space $M_{7,7} \subset C$. With increasing $\tau$, the point $\mathcal{E}(\tau)$ moves in $M_{7,7}$. The fact that we, observers, do not experience all of the spacetime $M_{1,3}$ or all of $M_{7,7}$ at once, is due to the localization of the wave packet. A further discussion of this topics can be found in Refs. [48, 28]. How it works for strings and branes, is discussed in [25]-[28]. The existing literature 28, 43, 44] shows that the Stueckelberg theory is a consistent physical theory, and that the quantization of the Stueckelberg field is not problematic at all.

Since the Stueckelberg theory admits tachyons, a question arises as to whether it is not in conflict with causality. Because tachyons in some reference frames are observed to move forward, and in some other frames to move backwards in the time coordinate $x^{0}$, the arrangements with tachyon emitters and absorbers are possible, such that they lead to causal loops in $M_{1,3}$. Since the latter space is a subspace of $C$, in most cases those loops will be merely projections of the lines in $C$ onto $M_{1,3}$, and hence not true loops in $M_{1,3}$. The $\tau$ and other coordinates of $C$ could be different, even if $x^{\mu}$ coincided. Hence, there would be no paradox, because no "change of history" would be possible in such a case. Thus, the problem would only be with the true loops in $C$, if they can occur. Then, one should take into account that what propagates, are not classical, sharply localized particles, but wave packets that always have a certain width. When such a wave packet of a causal loop arrives at the critical point in the past, a superposition of several possible histories would take place. There would be no causal paradox, but only a 'paradox' of several co-existing histories [28, 49, 50], which - according to the Everett many worlds interpretation [51, 52] of quantum mechanics - is not a paradox at all. In fact, the existence of causal loops, if experimentally established, would confirm the validity of the Everett interpretation of quantum mechanics, and disprove the other interpretations.

The same form of an ultrahyperbolic equation in $16 D$ Clifford space, $C$, holds for bradyons and tachyons. In order to experience a consistent evolution of localized fields, an observer must live on a suitable light-like hypersurface. All events on such hypersurface have the same value of the evolution parameter $\tau$. As the $\tau$ changes, so does the hypersurface. In other words, an observer is associated with a 1-parameter family of light-like hypersurfaces in $C$, distinguished by values of $\tau$. The families of hypersurfaces in $C$ that are not light-like cannot be associated with observers, because the Cauchy problem for such hypersurfaces 
is not well posed. An alternative possibility is that some observers are associated with space-like or time-like 4-surfaces on which the initial data for the Klein-Gordon field $\phi$ can be arbitrarily specified. Now the initial data for the bradyonic field, given on a space-like 4 -surface, include not only the derivative of $\phi$ with respect to one, but with respect to four time-like coordinates. For the tachyonic field, the initial data are given on a time-like 4 -surface, and include the derivatives of the field with respect to four space-like coordinates. Knowing such initial data, an observer can determine, although not uniquely, the behavior of the field outside the 4-surface. In our opinion, such indeterminism at the level of first quantized fields, should not be considered as problematic, because in a more complete, second quantized theory, fields are not well determined anyway.

The observed four-dimensionality of our spacetime implies that in any higherdimensional theory the extra dimensions must be either compactified or the observed matter must be localized on a four-dimensional surface in the higher-dimensional space. In the known examples where such compactification/localization occurs it requires highly nontrivial dynamical mechanisms that have been extensively studied in the literature. Any dynamical resolution of this issue must clearly lead to an effectively four-dimensional theory. That, according to the above, cannot be invariant under the superluminal boosts. In other words, any compactification/localizaton mechanism will break the invariance under the superluminal Lorentz transformations, rendering such transformations irrelevant in four dimensions. Such a reasoning would hold, if there would be only one four-dimensional surface, $M_{1,3}$, with observers observing the localized matter on $M_{1,3}$. However, the effective four-dimensional theory can be either in the spacetime $M_{1,3}$ of an observer $\mathcal{O}$, or in another spacetime $\tilde{M}_{1,3}$ of an observer $\tilde{\mathcal{O}}$. The two spacetimes, $M_{1,3}$ and $\tilde{M}_{1,3}$, are subspaces of the higher-dimensional spacetime that we started from, and can be transformed into each other by a superluminal boost in that higher-dimensional space. If for the higherdimensional space we take $M_{4,4}$ of Sec. 4 , with coordinates $\left(x^{\mu}, \tilde{x}^{\mu}\right), \mu=0,1,2,3$, then the subspace $M_{1,3}$ has coordinates $x^{\mu}=\left(x^{0}, x^{1}, x^{2}, x^{3}\right)$, whereas the subspace $\tilde{M}_{1,3}$ has coordinates $x^{0}, x^{1}, \tilde{x}^{2}, \tilde{x}^{3}$. Relative to $\mathcal{O}$, the observer $\tilde{\mathcal{O}}$ is tachyonic, and vice versa. Hence, bradyonic and tachyonic observers do not live in the same effective four-dimensional spacetime. The intersection of their respective spacetime is the two-dimensional spacetime, $M_{1,1}$, with coordinates $x^{0}, x^{1}$.

There is also a possibility that does not require a compactification of extra dimensions, or a localization of the observed matter. In the setup considered in Sec.4, we have a very special higher-dimensional space, namely the Clifford space, $C$. All the dimensions of $C$ are assumed to be observable even at the macroscopic scale, because those dimensions are associated with the position, size and orientation of the object. Therefore, the extra dimensions of $C$ can be large, and need not be compactified at sufficiently small scales, and be thus unobservable at macroscopic scales. If $C$ is curved, then we have the gravity in $C$. In order to reproduce, à la Kaluza-Klein, the usual, $4 D$ gravity in $M_{1,3}$ and the Yang-Mills interactions, there must be suitable isometries in $C$, given in terms of a set of 
Killing vector fields. We expect that such isometries arise dynamically in the presence of suitable extended sources, e.g., the branes in $C$ [27]. How precisely this works, remains to be investigated. The presence of isometries does not render the extra dimensions of $C$, i.e., the object's size and orientation, unobservable, it only makes the theory to be in agreement with the fact that effectively we have 4D gravity and Yang-Mills interactions.

\section{Conclusion}

We have shown that tachyons are not in conflict with special relativity and field theory, if those theories are properly extended. It is well-known that special relativity can be extended to encompass not only the subluminal, but also the superluminal reference frames, and the transformations relating those frames. Then it turns out that spacetime must be a complex 4-dimensional space, or a real 8-dimensional space with neutral signature $(4,4)$. The Klein-Gordon or the Dirac equation in such space is ultrahyperbolic, and thus problematic, regardless of whether the field is bradyonic or tachyonic. We have shown that the problem can be resolved if we take the Cauchy data not on a 7-dimensional hypersurface, but on a 4-dimensional surface that is space like for bradyonic and time like for tachyonic fields. The Klein-Gordon or the Dirac equation also is not problematic in the 16-dimensional Clifford space, $C$, where it can be written in the form of the corresponding generalized Stueckelberg like equation which describes localized propagating bradyonic and tachyonic fields.

Because bradyons in vacuum do not emit Čerenkov radiation, also tachyons of the extended relativity do not emit Cerenkov radiation in vacuum [7, 8]. This is a consequence of the postulated symmetry between bradyons and tachyons. The expectation that such radiation should accompany superluminal particles, is based on different theoretical as-

sumptions. Thus, according to the theory considered in this paper, no Čerenkov radiation in the form of electron-positron pairs is emitted by superluminal neutrinos.

\section{Acknowledgment}

This work was supported by the Slovenian Research Agency.

\section{References}

[1] T. Adam, et al. [OPERA Collaboration], Measurement of the neutrino velocity with the OPERA detector in the CNGS beam, arXiv:1109.4897 [hep-ex]

[2] M.P. Bilaniuk, V.K. Deshpande and E.C.G. Sudarshan, American Journ. Phys. 30 (1962), 718.

[3] G. Feinberg, Phys. Rev. 159 (1967), 1089.

[4] M.E. Arons and E.C.G. Sudarshan, Phys. Rev 173 (1968), 1622. 
[5] J. Dhar and E.C.G. Sudarshan, Phys. Rev. 174 (1968), 1808.

[6] M. Pavšič, The extended special theory of relativity, Preprint, 1971 (Unpublished) http://www-f1.ijs.si/ pavsic/ExtenRel71.pdf

[7] E. Recami and R. Mignani, Riv. Nuov. Cim.4 (1974), 209.

[8] E. Recami, Riv. Nuov. Cim. 9 (1986), 1.

[9] A.F. Antippa, Nuov. Cim. A 10 (1972), 389.

[10] W.A. Rodrigues, Jr. and J. Vaz, Jr., Subluminal and Superluminal electromagnetic Waves and the Lepton Mass Spectrum, arXiv hep-th/9607231.

[11] D. Shay and K.L. Miller, Nuov. Cim. A 38 (1977), 490.

[12] L. Robinett, Phys. Rev. D 18 (1978), 3610.

[13] R. Mignani and E. Recami, Lett. Nuov. Cim. 24 (1976), 171.

[14] E.A.B. Cole, Nuov. Cim. A 40 (1977), 171.

[15] E.A.B. Cole, J. Phys. A 13 (1980), 109.

[16] M. Pavšič, J. Phys. A 14 (1981), 3217.

[17] M. Tegmark, Class. Quant. Grav. 14 (1997), L69.

[18] C. Castro and M. Pavšič, Progress in Physics 1 (2005), 31.

[19] C. Castro and M. Pavšič, Phys. Lett. B 559 (2003), 74.

[20] W.M. Pezzaglia and A.W. Differ, Adv. Cliff. Alg. Proc. Suppl. 4(S1) (1994), 437.

[21] M. Pavšič, Phys. Lett. B 614 (2005), 85 hep-th/0412255.

[22] M. Pavšič, Int. J. Mod. Phys. A 21 (2006), 5905 gr-qc/0507053.

[23] S. Ansoldi, A. Aurilia, C. Castro, and E. Spallucci, Phys. Rev. D 64 (2001), 026003 hep-th/0105027.

[24] A. Aurilia, S. Ansoldi, and E. Spallucci, Class. Quant. Grav. 19 (2002), 3207.

[25] M. Pavšič, Found. Phys 33 (2003), 1277 gr-qc/0211085.

[26] M. Pavšič, Phys. Lett. B 692 (2010), 212 [arXiv:1005.1500 [hep-th]].

[27] M. Pavšič, Found. Phys. 37 (2007), 1197.

[28] M. Pavšič, The Landscape of Theoretical Physics: A Global View; From Point Particles to the Brane World and Beyond, in Search of a Unifying Principle, Kluwer, 2001.

[29] A. Chodos, A.I. Hauser, and V.A. Kosteleck'y, Phys. Lett. B 150 (1985), 431

[30] U.D. Jentschura and B.J. Wundt, From Generalized Dirac Equations to a Candidate for Dark Energy, arXiv:1205.0521 [hep-ph].

[31] U.D. Jentschura and B.J. Wundt, J. Phys. A 45 (2012), 444017 arXiv:1110.4171 [hep-ph]]. 
[32] A.O. Barut, Space-Like States in Relativistic Quantum Theory, in Tachyons, Monopoles, and Related Topics, Ed. E. Recami, North-Holland, 1978.

[33] V. Vyšín, Nuov. Cim. A 40 (1977), 113.

[34] V. Vyšín, Nuov. Cim. A 40 (1977), 125.

[35] http://wiki.physics.fsu.edu/wiki/index.php/Klein-Gordon_equation

[36] R. Mignani and E. Recami, Nuov. Cim. A 14 (1973), 169.

[37] B.S. Rajput and O.P.S. Cox, Phys. Lett. B 113 (1982), 183.

[38] V. Majernik, Found. Phys. 10 (1997), 357.

[39] M.C. Pant, P.S. Bisht, O.P.S. Negi, B.S. Rajput, Can. J. Phys. 78 (2000), 303.

[40] R. Courant and D. Hilbert, Methods of Mathematical Physics Vol. 1 and 2, Interscience, London, 1953

[41] D. Hestenes, Space-Time Algebra, Gordon and Breach, 1966.

[42] D. Hestenes and G. Sobczyk, Clifford Algebra to Geomteric Calculus, D.Reidel, 1984.

[43] J.R. Fanchi, Found. Phys. 23 (1993), 287, and many references therein.

[44] J.R. Fanchi, Parametrized Relativistic Quantum Theory Kluwer, 1993.

[45] U.D. Jentschura and B.J. Wundt, Eur. Phys. J. C 72 (2012), 1894 arXiv:1201.0359 [hep-ph]].

[46] T. Chang, A new Dirac-type equation for tachyonic neutrinos, arXiv:hep-th/0011087

[47] U.D. Jentschura, J. Mod. Phys. 3 (2012), 887.

[48] M. Pavšič M, Found. Phys. 21 (1991), 1005.

[49] M. Pavšič, Lett. Nuov. Cim. 30 (1981), 111.

[50] D. Deutsch, Phys. Rev. D 44 (1991), 3197.

[51] H. Everett III, Rev. Mod. Phys. 29 (1957), 454.

[52] H. Everett III, 1973 The theory of the universal wave function, in The Many-Worlds Interpretation of Quantum Mechanics ed. B.S. DeWitt and N. Graham, Princeton University Press, 1973. 